\title{
LA DINAMICA DE LA DESIGUALDAD EN CHILE: UNA MIRADA REGIONAL*
}

THE DYNAMICS OF INEQUALITY IN CHILE: A REGIONAL LOOK

\section{MICHELLE MIERES BREVIS**}

Universidad de Alicante

\begin{abstract}
Resumen
El estudio retrata la dinámica de las desigualdades en Chile (1990-2016) desde la perspectiva de sus regiones. Se construye una amplia base de datos con distintas medidas de desigualdad del ingreso. Se devela gran heterogeneidad entre las regiones chilenas. Se observa que las regiones más desiguales, son igualmente aquellas con menor PIB per cápita y desarrollo. A nivel geográfico, suelen ser las regiones del sur las perdedoras en materia de desarrollo y desigualdad. Se demuestra convergencia regional en términos de PIB per cápita y de desigualdad del ingreso, a pesar de la persistencia de esta a través de los años.
\end{abstract}

Palabras clave: Desigualdad, crecimiento económico, crecimiento regional, desarrollo económico, convergencia.

Clasificación: D31, D63, F43, O11.

* Este artículo es parte de mi trabajo de investigación en el programa de Doctorado Interuniver sitario DEcIDE, en la Universidad de Alicante (Carretera de San Vicente del Raspeig, s/n, 03690 San Vicente del Raspeig, Alicante, España. Teléfono: 96590 3609). El trabajo se ha beneficiado de los comentarios y sugerencias de mi directora de tesis María Dolores Guilló y de los asistentes al Seminario Novel 2018, realizado en la Universidad Miguel Hernández de Elche, en el cual presenté este estudio. El trabajo también fue expuesto en el Seminario "Miradas de la Desigualdad en Chile y La Araucanía", en la Universidad de La Frontera, Temuco-Chile. Correo: mmb124@alu.ua.es

** Este trabajo es posible gracias al apoyo de CONICYT PFCHA/DOCTORADO BECAS CHILE/2017 $-72180047$. 


\begin{abstract}
The study shows the dynamics of inequalities in Chile (1990-2016) from a regional perspective. A broad database is constructed including different measures of income inequality, revealing the enormus heterogeneity across the Chilean regions. The data shown that the most unequal regions are also those with the lowest GDP per capita and other development indicators. At the geographical level, the southern regions are the losers in terms of development and inequality. It is shown that there exists regional convergence in terms of GDP per capita and in the Gini coefficient, despite the high persistence in inequality over the period.
\end{abstract}

Keywords: Inequality, economic growth, regional growth, economic development, convergence.

JEL Classification: D31, D63, F43, 011 .

\title{
1. INTRODUCCION
}

No se puede negar que Chile ha experimentado un desarrollo sin precedentes desde el retorno a la democracia (1990). Se ha logrado en un escenario de estabilidad política y social, preservando equilibrios macroeconómicos a pesar de los shocks internacionales (Sunkel e Infante, 2009). Asimismo, las políticas sociales, enfocadas a la población de menores ingresos han obtenido buenos resultados disminuyendo la pobreza del país y en cada una de sus regiones. Chile fue el primer país Latinoamericano invitado a ser parte de la Organización para la Cooperación y el Desarrollo Económico (OCDE). No obstante, existe una tarea pendiente, que es la desigualdad. Actualmente, Chile es el segundo país más desigual de la OCDE (índice de Gini antes y después de impuestos y transferencias). La desigualdad de Chile es intra e interregional, los ingresos del $20 \%$ de la población más rica son 10 veces mayores que los del quintil más pobre (OCDE, 2018), siendo sorprendentemente mayor que el promedio de los países de la OCDE.

Este artículo tiene por objetivo retratar las desigualdades a nivel socioeconómico en Chile, durante el período 1990-2016, desde la perspectiva de sus regiones. Para lograr este objetivo, la segunda sección da una mirada global al territorio chileno. A continuación (sección 3), se estudia el crecimiento económico de Chile; se presentan los principales indicadores de pobreza y se analiza la evolución de la desigualdad del ingreso. La sección 4 expone los indicadores de desarrollo humano y regional, y entrega un retrato de las principales desigualdades a nivel social. Los resultados del análisis de convergencia del PIB per cápita y la desigualdad están en la sección 5. Finalmente se presentan las principales conclusiones del trabajo. 
La relevancia de este estudio es la construcción de una gran base de datos a nivel regional, durante un largo período de tiempo. A los datos ya disponibles en distintas fuentes secundarias, se incorpora el cálculo de indicadores como el índice de Gini regional (2013 y 2015 bajo la metodología nueva y antigua); los ratios 10/10 y 20/20 (1990-2015) y el PIB per cápita regional (1990-2016). Los dos primeros indicadores se calculan con base en la encuesta CASEN. Para el PIB per cápita regional se utilizan distintas bases dispuestas por el Banco Central de Chile y el INE, logrando construir una única base amplia y comparable en el tiempo, utilizando el método de tasa de variación (Correa et al., 2002). Todo ello ha permitido realizar este retrato y poder plasmar las desigualdades regionales, que a la fecha aquejan al país. Este trabajo es un esfuerzo necesario para la sociedad chilena, de reflexionar sobre el tamaño de las brechas que nos separan. Conocer la heterogeneidad del territorio chileno, las regiones más y menos vulnerables y el comportamiento de los distintos indicadores de desigualdad en el tiempo, es un primer paso para hacer políticas públicas y obtener el anhelado crecimiento y desarrollo inclusivos.

La metodología de investigación consiste en un análisis descriptivo y comparativo de las regiones del país, así como de un análisis de convergencia regional. La información recopilada para el desarrollo de este trabajo, se ha obtenido de fuentes secundarias, desde distintas bases de datos mundiales y nacionales. A nivel país se utiliza principalmente, la Encuesta de Caracterización Socioeconómica Nacional (CASEN) del Ministerio de Desarrollo Social y datos de productividad del Banco Central de Chile. Asimismo, se contacta con diferentes instituciones, como el Instituto Nacional de Estadísticas, al que se solicita datos antiguos poblacionales y vitales no disponibles en la web ${ }^{1}$.

Las principales conclusiones de este trabajo, revelan la gran heterogeneidad que existe entre las regiones de Chile, siendo consistente con los trabajos de Agostini y Brown (2007; 2010), Ramírez et al. (2009) y Mac Clure y Calvo (2013), quienes avalan la importancia de trabajar a distintas escalas territoriales ${ }^{2}$. Se observa que las regiones más desiguales a nivel social y económico, son igualmente aquellas con menor PIB y desarrollo económico. Además, a nivel geográfico, suelen ser las regiones del sur del país las perdedoras en materia de desarrollo y desigualdad.

A través de un análisis de convergencia, se concluye que en Chile, las regiones más pobres y desiguales, tienden a crecer más que aquellas más ricas y equitativas. Es decir, las regiones más perjudicadas convergen buscando alcanzar a los territorios más favorecidos del país. Lo que marca la desigualdad en Chile, es el aún elevado nivel de desigualdad y su persistencia, a pesar de la mencionada convergencia.

1 La tarea de recopilación de datos no fue fácil, sobre todo al querer abordar el largo plazo, sin embargo, se debe agradecer a las distintas instituciones que cooperaron entregando información a través del Portal de Transparencia del Estado.

2 Estos autores hacen especial énfasis en la importancia de trabajar a nivel subregional. Mac Clure y Calvo (2013) proponen una división administrativa basada en el actual estado del proceso de urbanización. 
"Obtener una imagen más clara de la pobreza y la privación es un primer paso fundamental para elaborar y aplicar políticas e intervenciones más eficaces y para orientar mejor los escasos recursos hacia donde tengan una mayor repercusión" (Melinda Gates, Copresidenta de la Fundación Bill y Melinda Gates).

\section{CONTEXTO NACIONAL}

Esta sección busca contextualizar el trabajo, dando una mirada global al país de estudio, Chile, presentando algunas de sus principales características sociodemográficas, contando un poco de su historia política y su evolución a nivel económico.

Chile es un país largo y angosto, ubicado al sudoeste de América Latina, limita al norte con Perú, al noreste con Bolivia y al este con Argentina. De acuerdo con la Encuesta de Caracterización Socioeconómica Nacional (CASEN) de 2015, el país tiene una población de más de 17,5 millones de personas ${ }^{3}$, de las cuales un $87 \%$ vive en zonas urbanas. En Chile, la ley reconoce la existencia de nueve pueblos originarios ${ }^{4} \mathrm{y}$ de acuerdo con la CASEN (2015) solo el 9\% de la población chilena declara pertenecer a una etnia, siendo mayormente Mapuches (84\% del total). Las regiones con mayor porcentaje de población indígena son la Región Metropolitana y La Araucanía, siendo en esta última donde la mayoría de ellos vive en zonas rurales (59\%).

El país está conformado por 16 regiones $^{5}$, siendo la capital nacional la ciudad de Santiago en la Región Metropolitana. El territorio chileno es abundante en recursos naturales, es el mayor productor de cobre a nivel mundial y tiene un importante rol dentro de los países exportadores de productos agrícolas, forestales y ganaderos (OCDE y CEPAL, 2016).

Chile es un país centralizado, condición que se remonta al siglo XIX tras la Constitución de 1833, luego de la guerra civil de 1829, donde los conservadores triunfan, imponiendo un régimen unitario y centralista (Montecinos, 2005). De acuerdo con Palma (2009), existieron tres olas centralizadoras en Chile durante el siglo XX, siendo la tercera de ellas en la época del Gobierno militar (1973-1989) y durante los Gobiernos de la concertación (partidos por la democracia) que siguieron. Los Gobiernos de la época buscaban el desarrollo económico, principalmente fomentando la apertura a los mercados extranjeros, y se consideraba al mercado como el mejor asignador de los recursos. A finales del siglo XX, principios del XXI, se amplía la brecha entre la

3 Para que un análisis basado en encuestas de hogares con diseño muestral probabilístico tenga validez sobre la población objetivo, se debe usar un ponderador para la estimación de cualquier variable de interés, lo que da cuenta del número de personas de la población que representa un individuo encuestado. El ponderador se denomina factor de expansión. Para este estudio se utiliza el factor de expansión regional, puesto a disposición por el Ministerio de Desarrollo Social, responsable de la Encuesta CASEN.

4 Aimara, Rapa-Nui, Quechua, Mapuche, Atacameño, Collas, Alacalufes, Yagán y Diaguita.

5 La región número 16, Ñuble, se incorpora a partir de 2018, separándose de la región del Biobío. 
Región Metropolitana y el resto de las regiones, sobre todo en materia de inversión, gasto público, consumo, entre otros (Palma, 2009). Al finalizar la dictadura militar, en el año 1989, y con la elección popular del ex-presidente Patricio Aylwin (concertación de partidos por la democracia), se suscitaron una serie de cambios políticos y sociales en el país. Este Gobierno y los que le siguieron, tenían como principal objetivo la transición a la democracia, así como el fomento de un mayor desarrollo económico territorial.

En marzo del año 2010, bajo el segundo mandato de la presidente Michelle Bachellet, Chile se convirtió en el miembro número 31 de la Organización para la Cooperación y el Desarrollo Económico (OCDE) y el primero a nivel Sudamericano. En su discurso, Angel Gurría (Secretario General de la OCDE), declara que la experiencia chilena enriquecerá el acervo de la OCDE en cuestiones clave, siendo un ejemplo al combinar crecimiento económico y mayor bienestar social. Gurría comentó que Chile ocuparía un lugar que le pertenece por mérito propio, cuyo objetivo es que comparta sus buenas prácticas y políticas públicas con los demás países de la OCDE. Además enfatizó que Chile sería parte de este conjunto de países que ayudan a que la economía mundial sea más fuerte, limpia y justa (informa oecd.org, el día 11 de Enero de 2010, a través del titular "Chile, primer país sudamericano miembro de la OCDE").

Durante los últimos 35 años, Chile ha tenido un crecimiento económico sostenido gracias al alza en los precios de las materias primas y a las políticas macroeconómicas. Se ha invertido en infraestructura y distintos servicios, incluidos medioambientales, inversiones que han tenido repercusiones en el bienestar de las personas y en la generación de mayor empleo. De acuerdo con el Banco Mundial (2017), durante las últimas décadas Chile ha sido una de las economías Latinoamericanas de más rápido crecimiento, lo que le ha permitido reducir sus niveles de pobreza de un $26 \%$ a un 7,9\% en el período 2000-2015. Sin embargo, los buenos indicadores macroeconómicos no han podido mitigar el efecto de la persistente desigualdad de ingresos chilena. En el informe económico de la OCDE (2012) se reconoce que la pobreza y desigualdad son elevadas en Chile, comparadas con los demás miembros de la OCDE, siendo explicadas en parte, por la poca capacidad redistributiva de los impuestos del país.

El informe económico de la OCDE (2013) concluye que el fuerte crecimiento de Chile ha traído consecuencias para el ecosistema, uno de ellos es el mayor gasto energético. Si bien existe la conciencia a nivel público y privado del costo medioambiental, los esfuerzos para cubrir las externalidades negativas, a través de nuevas tecnologías e impuestos energéticos, no han sido suficientes (OCDE, 2013). A similares resultados llega el estudio de la OCDE y CEPAL (2016), el cual informa que en las últimas décadas, el país, además del ya mencionado crecimiento, ha mejorado el acceso de la población a servicios claves como tratamiento de aguas residuales y transporte público urbano, sin embargo, estas mejorías se vieron acompañadas de una persistente desigualdad del ingreso y daños medioambientales.

La desigualdad es un tema que aflora en cada uno de los informes económicos que la OCDE realiza para Chile. En 2015, el país ocupa el puesto número dos en desigualdad 
entre los miembros de la OCDE (medida a través del índice de Gini antes y después de impuestos y transferencias), después de México. Lo siguen Turquía, Estados Unidos y Lituania, siendo los países con menor desigualdad Islandia, Eslovaquia y Eslovenia (OCDE, 2018). La desigualdad de Chile es intra e interregional, los ingresos del 10\% de la población más rica del país son 27 veces mayores que el decil más pobre (OCDE, $2015)^{6}$, por sobre el promedio de los países de la OCDE, donde el $10 \%$ más rico gana 9,6 veces más que el $10 \%$ más pobre (siendo esta la mayor diferencia de los últimos 30 años) (Keeley, 2015). Actualmente en Chile, el 20\% de mayores ingresos lo es 10 veces más que el $20 \%$ más pobre (OCDE, 2018).

Pero no solo existe una concentración económica en Chile, sino también política, poblacional, de los recursos y del sector industrial. En consecuencia, el país se encuentra en una época de constantes protestas sociales de diversa índole y es cada vez más frecuente que las regiones se manifiesten en contra de un Gobierno central, que no ha sido capaz de satisfacer las necesidades de un territorio tan heterogéneo como Chile (Mieres, 2015). Es imperativo que la distribución de la riqueza en Chile sea más equitativa; que el crecimiento económico sea más inclusivo y que se facilite la movilidad económica y social de la población chilena. Para alcanzar este mayor nivel de desarrollo y por ende el mejoramiento de la calidad de vida de la población, es necesario que el proceso hacia la equidad considere las diferencias territoriales del país.

\section{CRECIMIENTO, POBREZA Y DESIGUALDAD DEL INGRESO}

Este trabajo expone un retrato de la dinámica de la desigualdad socioeconómica en Chile. Esta sección, en una primera instancia, estudia el crecimiento económico de Chile y expone los principales indicadores de pobreza. En una segunda parte, realiza un análisis de diferentes indicadores de la desigualdad del ingreso a nivel país y regional. Se devela la dinámica de estos indicadores en el tiempo y la heterogeneidad de la desigualdad entre los distintos territorios. Se verá que, en el período analizado, todas las regiones del país redujeron su nivel de pobreza, lo que se reflejó en los buenos resultados a nivel país. Sin embargo, las desigualdades se han mantenido casi invariantes, sobre todo en el último período (2013-2015), donde incluso siete de las 15 regiones aumentaron el índice de Gini. Esta sección trabaja, a nivel internacional, con la base de datos de Summers y Heston (Penn World Table). A nivel nacional, se obtiene el PIB per cápita a partir de la renta informada por el Banco Central de Chile y el INE. La Encuesta CASEN permite mostrar datos de pobreza por ingreso y multidimensional, así como presentar los distintos indicadores de desigualdad en el tiempo.

6 El informe expone datos de 2012 o los últimos encontrados para cada país. 


\subsection{Crecimiento del PIB y dinámica de la pobreza en Chile}

El PIB per cápita ha sido ampliamente estudiado en la literatura que relaciona el crecimiento económico con la desigualdad de un territorio. Comenzando por Kuznets (1955), cuya teoría sostiene que cuando una economía comienza a crecer, también lo hacen las disparidades, hasta llegar a un cierto punto de desarrollo, donde a pesar del mayor crecimiento económico, las desigualdades van disminuyendo, tomando la forma de una U invertida. Por su lado, la Nueva Geografía Económica plantea que las desigualdades entre regiones pueden mantenerse en el tiempo e incluso crecer junto con la economía de un país, al existir un centro que aprovecha los mayores beneficios del crecimiento, mientras que las regiones de la periferia pierden esa oportunidad (Merchand, 2009).

De lo anterior, la importancia de conocer la dinámica productiva de Chile y sus diferencias regionales. Acorde con el Banco Mundial, el PIB real de Chile para el año 2016, fue de aproximadamente 145.957 mil millones de pesos chilenos. A nivel per cápita esto equivale a $\$ 8.149 .596$ al año ${ }^{7}$.

Para exponer la situación de Chile en el mundo, la Tabla 1 muestra una comparación con los países de mayor y menor PIB per cápita. Países de Asia y Europa ocupan los primeros lugares ${ }^{8}$. En los últimos puestos se encuentran países del continente africano, donde el PIB por persona más bajo corresponde a la República Centroafricana, siendo tan solo 0,03 veces el de Chile.

TABLA 1

PAISES CON MAYOR Y MENOR PIB PER CAPITA 2014

\begin{tabular}{|l|c|c|}
\hline \multicolumn{1}{|c|}{ País } & $\begin{array}{c}\text { PIB per cápita a PPA } \\
\text { (US\$ 2011) }\end{array}$ & País v/s Chile \\
\hline Catar & 151.763 & 7,12 \\
Noruega & 78.293 & 3,67 \\
Emiratos Arabes Unidos & 70.096 & 3,29 \\
Chile & 21.317 & 1 \\
Liberia & 870 & 0,04 \\
Burundi & 842 & 0,04 \\
República Centroafricana & 600 & 0,03 \\
\hline
\end{tabular}

Fuente: Elaboración propia, datos Summers y Heston (PWT) (2018).

7 Equivalente a 20.913 dólares per cápita en PPA (2016), anuales.

8 Estados Unidos por su parte, obtiene el puesto número diez, con un PIB per cápita 2,4 veces superior al chileno. 
A nivel Latinoamericano, Chile lidera en el territorio, con un PIB per cápita que es 12 veces mayor al país con el peor resultado, Haití. Sus más cercanos competidores son Panamá y Argentina. Panamá, de acuerdo con el Fondo Monetario Internacional (FMI, 2017), es una economía emergente con gran posibilidad de mantenerse a la cabeza de este grupo en los próximos años.

En el largo plazo Chile demuestra un importante desempeño económico. Realizando una comparación con todos los países miembros de la OCDE, Chile destaca con un crecimiento promedio del PIB per cápita de 4,6\% en el período 1990-2014, siendo superado solo por Polonia e Irlanda. El Gráfico 1(a) considera en el eje de las abscisas el PIB per cápita al comienzo del período, y en las ordenadas la tasa de crecimiento promedio del PIB per cápita. Se observa una correlación negativa, la tendencia es que los países más pobres tienden a crecer más a prisa que los países más ricos de 1990 ( $\beta$ negativo y estadísticamente significativo al 95\% de confianza). El Gráfico 1(b) muestra la posición de cada país en 2014, respecto del año 1990; se observa que Chile ha logrado alcanzar a México y Turquía, pasando de un PIB per cápita de 7.247 US\$ (a PPA de 2011) a 21.317 US\$ en 24 años, dejando el último puesto como país de menores ingresos por habitante de la OCDE.

A nivel regional, en el Gráfico 2 se observan las diferencias de ingresos per cápita existente en el territorio 9 . A través del tiempo, solo cinco regiones se encuentran sobre el promedio nacional, siendo tres regiones de la zona norte del país, Antofagasta, Atacama y Tarapacá (para este efecto incluye los resultados de Arica y Parinacota y la región de Tarapacá), más Magallanes y la Región Metropolitana. Las regiones relegadas son La Araucanía, el Maule y la región de Los Lagos (que incluye la región de los Ríos). En el último año, las regiones con mayor aporte del sector silvoagropecuario y pesquero al PIB regional son: Aysén, el Maule y O’Higgins, mientras que las regiones con mayor actividad minera son Antofagasta, Atacama y Tarapacá (ver Anexo A). Siguiendo a Sunkel e Infante (2009), la desigualdad está marcada no solo por las diferencias en ingreso y acumulación de riqueza, sino también por la heterogeneidad de su estructura productiva ${ }^{10}$.

Respecto a la tasa de crecimiento, en 2016, La Araucanía es la región con mayor crecimiento a nivel real, a pesar de los bajos indicadores socioeconómicos que se darán a conocer más adelante. Mientras tanto, la menor variación se observó en el norte, en la región de Antofagasta, cuyo PIB disminuyó un 2,9\% respecto al año anterior, sin embargo, sigue siendo una de las regiones que más aporta al PIB nacional $(9,7 \%)$, gracias al producto minero de esta zona. La Región Metropolitana es por excelencia la región que más produce en el país, por sí sola aporta el 42\% al PIB nacional, sus

9 Estos datos están validados por el Banco Mundial, ya que en su Base de Datos oficial se utilizan los mismos datos aportados por el Banco Central de Chile.

10 Esto ya que en el país conviven empresas transnacionales exportadoras, con altas rentabilidades, junto con la pequeña y mediana empresa y microempresas informales de baja productividad, que se concentran en el mercado local y con escasos vínculos con la gran empresa. 


\section{GRAFICO 1}

PIB PER CAPITA (MILES DE US\$2011), PAISES OCDE

(a) PIB per cápita 1990/Crecimiento promedio.

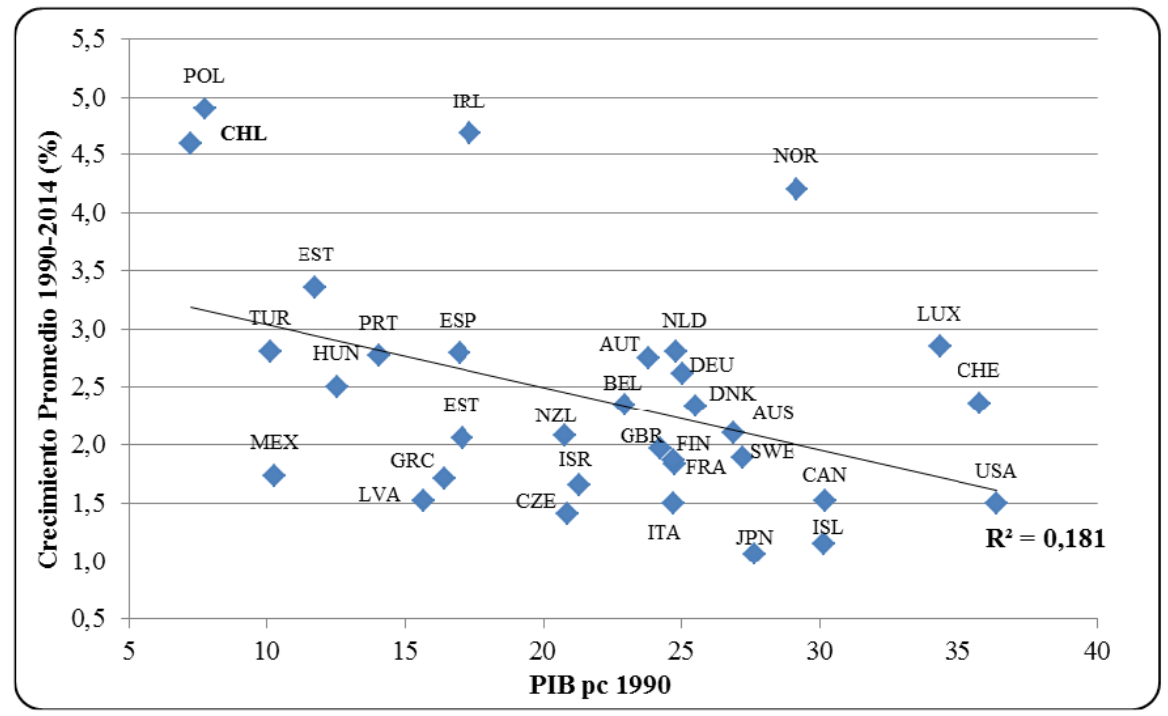

(b) PIB per cápita 1990/2014.

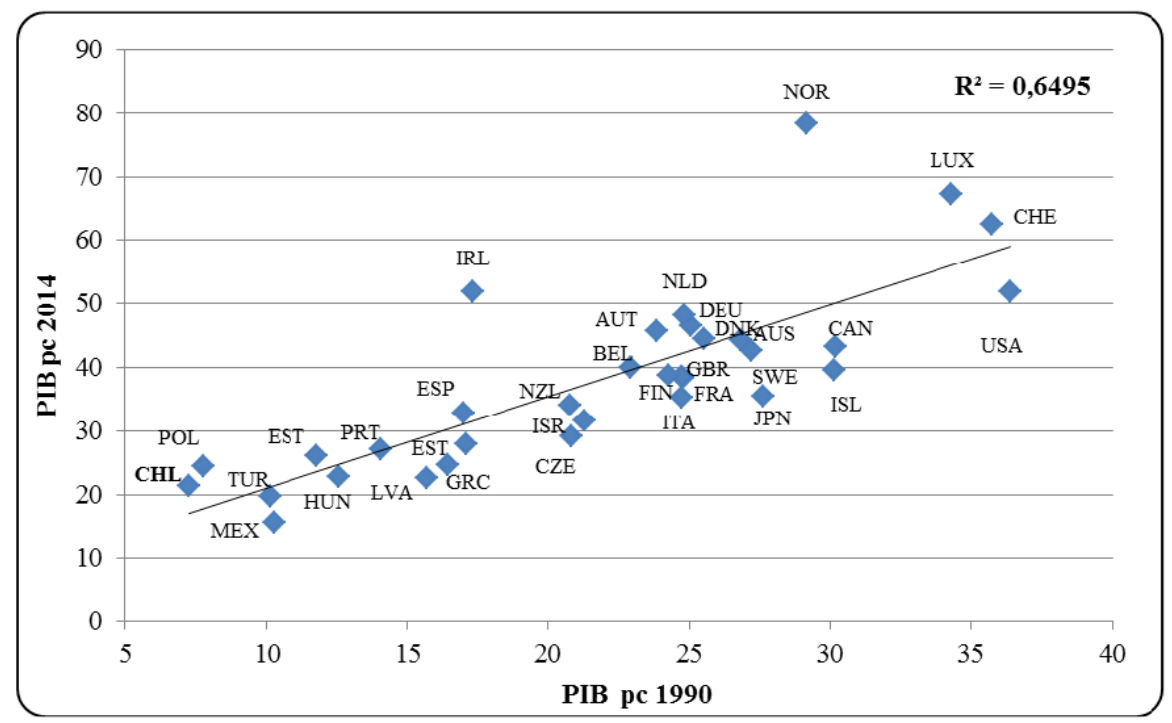

Fuente: Elaboración propia, datos PWT. 
principales ingresos provienen del sector de servicios financieros y empresariales y del comercio (Ver Anexo B).

\section{GRAFICO 2}

LN(PIB PER CAPITA) REGIONES DE CHILE (CLP REF. 2013), 1990-2016

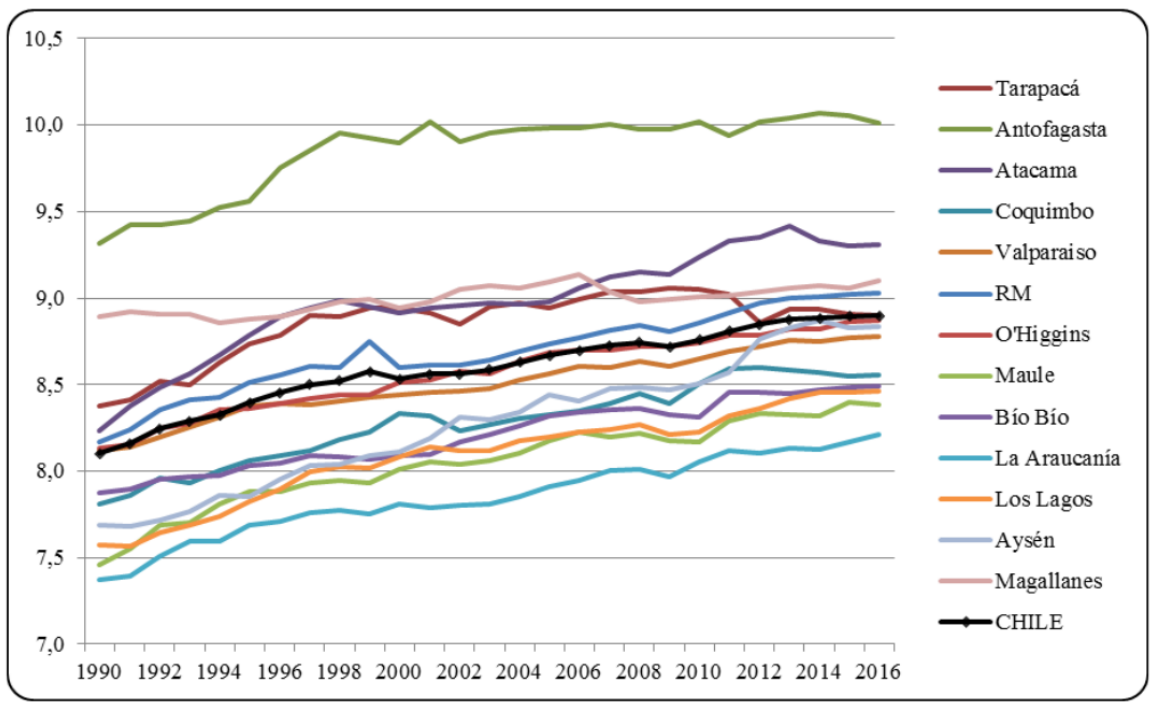

Fuente: Elaboración propia datos BC.

A continuación esta sección analiza la pobreza en Chile, un fenómeno complejo de definir y medir, entendido como una condición privativa, en la cual las personas carecen de recursos suficientes para satisfacer sus necesidades básicas como vivienda, alimentación, vestuario o salud. Además, es un término asociado a otros fenómenos negativos, como la desigualdad y la falta de oportunidades, afectando el bienestar de la población (Ministerio de Desarrollo Social, 2011). En la literatura económica, desde hace décadas, existe consenso en que un país o territorio no debe ser evaluado únicamente por sus indicadores económicos de productividad, sino que también por su capacidad para reducir la pobreza y la desigualdad, ambos conceptos entendidos como males para una sociedad (Gasparini et al., 2014). Al ser la pobreza reconocida como mal social, no es de extrañar que sea un concepto ampliamente estudiado y que sea propósito de cada Gobierno reducirla al máximo. Asimismo, el hecho de obtener un ingreso insuficiente es consecuencia de las desigualdades estructurales de un sistema económico (Macías, 2014). 
Para reducir la pobreza que aún afecta a Chile, así como la desigualdad, se requiere de buenos instrumentos que puedan identificar, cuantificar y caracterizar, de una forma precisa, a la población más vulnerable del país, de manera de poder aplicar la política pública eficientemente. Bajo este criterio, el Ministerio de Desarrollo Social, encargado de la Encuesta CASEN, a partir del año 2013 utiliza una nueva metodología de medición de Pobreza por Ingresos, tomando en cuenta los nuevos patrones de consumo chilenos e imponiendo estándares más altos que se adapten al actual nivel de desarrollo del país. Asimismo, incorpora la medición de la Pobreza Multidimensional, siguiendo el ejemplo de países más desarrollados.

La pobreza multidimensional en Chile consideraba originalmente cuatro dimensiones: educación, salud, trabajo y seguridad social y vivienda. A partir del año 2015 se incorpora redes y cohesión social, y la dimensión vivienda se amplía a vivienda y entorno. Para este año se estimó que en Chile, el 11,7\% de la población se encontraba en situación de pobreza por ingresos $(8,1 \%$ pobreza no extrema y $3,5 \%$ pobreza extrema) y un 20,9\% en situación de pobreza multidimensional (Ministerio de Desarrollo Social, 2015c). Los Gráficos 3 y 4 demuestran una evolución positiva del porcentaje de población en situación de pobreza, tanto por ingresos como multidimensional. La mayor variación se observa en la población pobre extrema, pasando de un $12,6 \%$ en 2006 a un $3,5 \%$ en 2015.

\section{GRAFICO 3}

POBLACION EN SITUACION DE POBREZA Y POBREZA EXTREMA POR INGRESOS (\%), 2006-2015

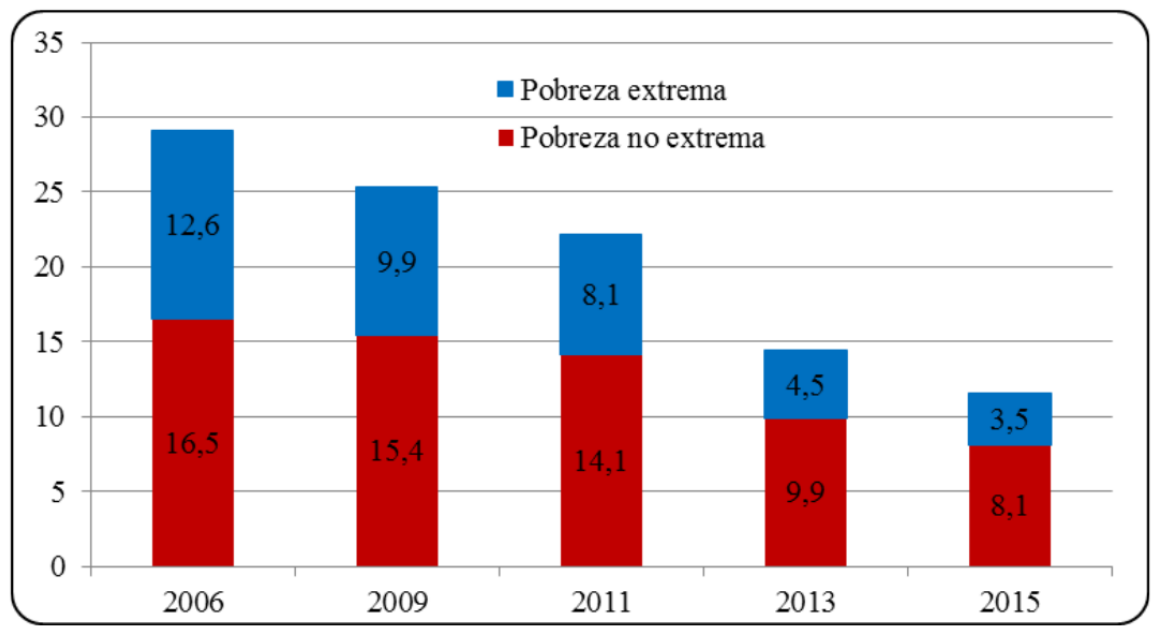

Fuente: Adaptada de Ministerio de Desarrollo Social (2015c). 
El Gráfico 4 deja ver cómo incorporar una nueva dimensión acrecienta el porcentaje de población en situación de pobreza en 1,8 puntos porcentuales. Si bien no es comparable con años anteriores, entrega un resultado más cercano a la realidad, ya que esta es una variable de gran impacto en la calidad de vida de la población chilena. La pobreza multidimensional en 2015 se vio afectada principalmente de las dimensiones trabajo y seguridad social y educación $(31,1$ y $25,8 \%$ respectivamente).

\section{GRAFICO 4}

POBLACION EN SITUACION DE POBREZA MULTIDIMENSIONAL (\%) 2009-2015

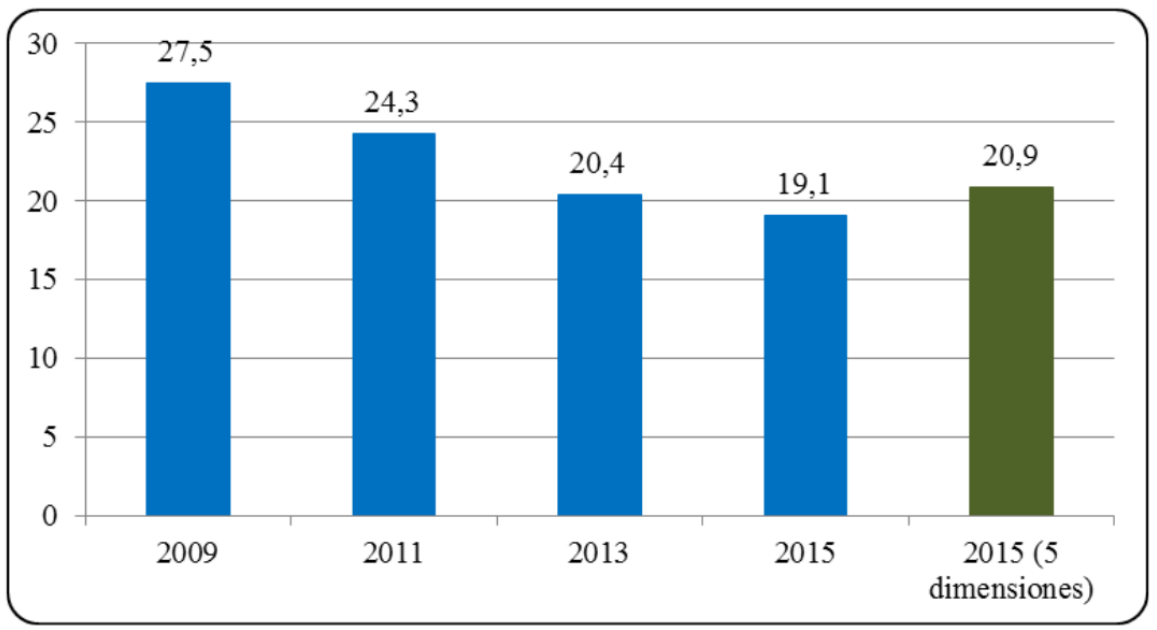

Fuente: Adaptada de Ministerio de Desarrollo Social (2015c).

A nivel territorial, el Gráfico 5 expone las diferencias interregionales en ambos tipos de pobreza. Las regiones con menos personas en situación de pobreza, tanto por ingresos, como multidimensional son Magallanes, Antofagasta y Aysén. Las regiones con mayor pobreza por ingresos son La Araucanía, Maule y la región del Biobío. Al considerar la pobreza multidimensional, nuevamente la región de La Araucanía es la más vulnerable, pero en este caso seguida por dos regiones del norte del país, Atacama y Coquimbo. Solo cinco de las 15 regiones se encuentran por debajo del promedio nacional evaluando ambos tipos de pobreza (Magallanes, Aysén, la Región Metropolitana, Antofagasta y Tarapacá). Por su lado, La Araucanía sobrepasa los promedios nacionales en ambos casos, y se observa que la diferencia entre ambos tipos de pobreza no es tan amplio, por lo que se infiere que el ingreso es la mayor 
causa de pobreza en este territorio, lo que se avala con el resultado del PIB per cápita expuesto anteriormente.

Al revisar la evolución de la pobreza multidimensional (cuatro dimensiones), se aprecia que la dinámica es distinta en cada región, sin embargo, a lo largo de los años han sido invariables los nombres de las regiones con más y menos pobreza, siendo para todos los años La Araucanía y Magallanes, respectivamente. Destacando esta última región dentro del grupo con mayor disminución de la pobreza entre 20092015, siguiendo muy de cerca a la región de Aysén, la cual con un 51,5\% es la que ha obtenido el mejor progreso. La menor evolución, muy por debajo del promedio nacional, se observa en Arica y Parinacota y en la región de O’Higgins (Ver Anexo C).

\section{GRAFICO 5}

PERSONAS EN SITUACION DE POBREZA Y POBREZA MULTIDIMENSIONAL (CON ENTORNO Y REDES) 2015

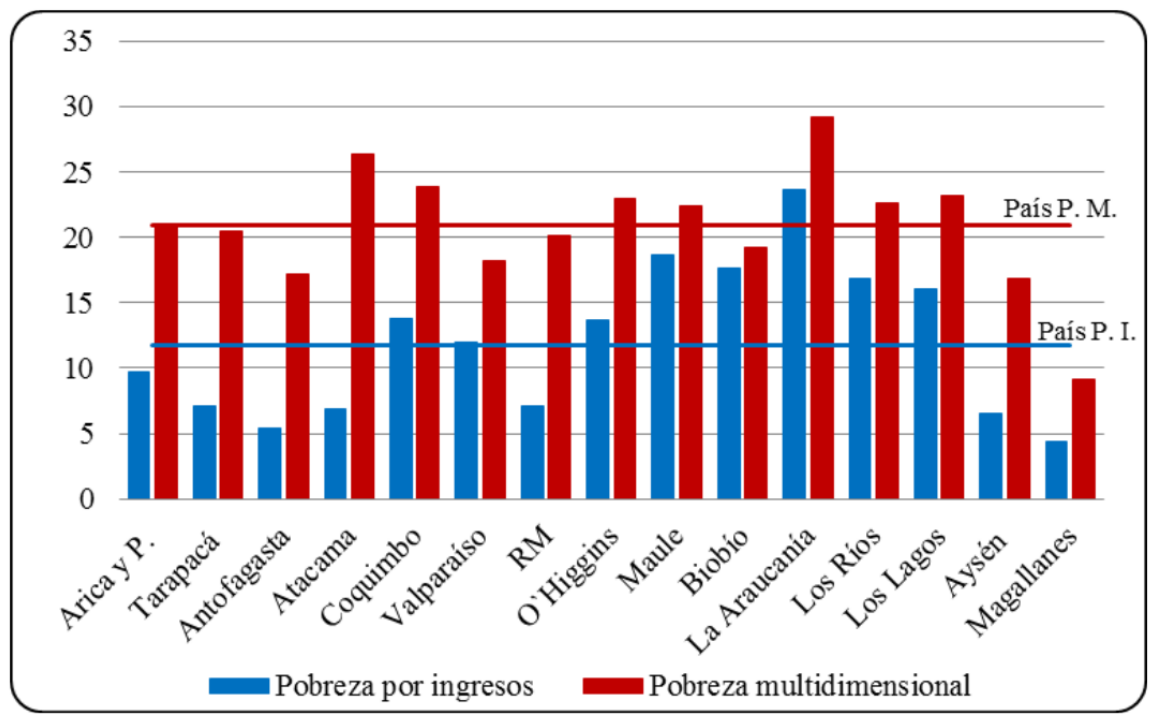

Fuente: Elaboración propia en base a Ministerio de Desarrollo Social (2015c).

\subsection{Evolución de la Desigualdad del Ingreso en Chile}

Los buenos indicadores a nivel de crecimiento económico de las últimas décadas en Chile, no han podido mitigar la persistente desigualdad del ingreso. Anteriormente, Soto y Torche (2004) concluyen lo mismo, demostrando que, entre 1975 y 2000, el PIB 
per cápita chileno creció un 5\% al año, sin embargo, las regiones no se beneficiaron de igual forma. La pobreza cayó significativamente en todas ellas, pero la desigualdad del ingreso se mantuvo constante. Por lo tanto, estudiar solo el comportamiento nacional no es un análisis completo, ya que oculta cambios significativos a nivel regional. Conocer la dinámica de desigualdad regional será un punto importante en el diseño de políticas públicas y estrategias de desarrollo diferenciadas, muy necesarias en un país con regiones tan heterogéneas como Chile.

Agostini y Brown $(2007 ; 2010)$ realizaron un estudio a nivel desagregado de las desigualdades chilenas, logrando obtener un índice de Gini comunal ${ }^{11}$. Los resultados confirmaron la mencionada heterogeneidad del territorio chileno, obteniendo Ginis que van de 0,41 a 0,63. Asimismo, se expone que las regiones del sur, así como la Metropolitana, tienden a tener comunas con mayor nivel de desigualdad del ingreso (similares resultados obtuvieron Ramírez et al. [2009] en su estudio comunal).

En el período 1990 y 2013, la desigualdad del ingreso en Chile ${ }^{12}$, medida a través del índice de Gini, evolucionó de una forma similar a una U invertida (ver Gráfico 6), crece a partir de 1992 para luego descender desde 2003 y de manera constante desde 2009. Bajo la metodología tradicional Chile pasó de un índice de Gini de 0,57 en 1990 a 0,53 en 2013. La nueva metodología ${ }^{13}$ da como resultado un índice menor, posiblemente porque las familias más vulnerables suelen ser más numerosas, luego al evaluar la distribución del ingreso total de los hogares, es esperable que sea más igualitario que al medirlo a nivel per cápita. A pesar de esta diferencia, se observa el mismo comportamiento a la baja. En los últimos años la reducción de la desigualdad ha sido mínima, llegando a ser el índice de 0,495 en 2015.

Otro indicador de desigualdad es el índice10/10, que expresa el ingreso del 10\% más rico en función del 10\% más pobre de la población. Para ello, la Tabla 2 presenta el promedio del ingreso autónomo del hogar (ver definición en Anexo E) por deciles, de acuerdo a la Encuesta CASEN de 2015. Los resultados denotan grandes inequidades del ingreso promedio. Observando los extremos se evidencia que el 10\% de los hogares de mayores ingresos son 27,2 veces más ricos que el decil de menores ingresos. El resultado es aún más abismante si consideramos los ingresos autónomos per cápita del hogar, donde la razón 10/10 aumenta a 45,1. Lo anterior no es de extrañar, ya que como se señaló en la Sección 2, Chile es el segundo país más desigual de entre los países miembros de la OCDE.

11 Utilizan dos bases de datos, la Encuesta Nacional de Caracterización Socioeconómica (Casen) de 2003 y el Censo de Población y Vivienda de 2002.

12 Los cambios metodológicos de la Encuesta CASEN aplicados a partir del año 2013 tuvieron también un impacto en el cálculo de los indicadores de desigualdad de ingresos. La nueva metodología se aplicó a bases de datos solo desde el año 2006. Para entender la evolución de la desigualdad a largo plazo, se expondrán también los resultados del índice de Gini bajo la metodología tradicional.

13 En ambas metodologías se utiliza el ingreso autónomo para el cálculo del índice de Gini. La nueva metodología, a diferencia de la anterior utiliza el hogar como unidad de análisis (no las personas) y se dejan de considerar los individuos que no reportan ingreso alguno. 


\section{GRAFICO 6}

EVOLUCION INDICE DE GINI EN CHILE

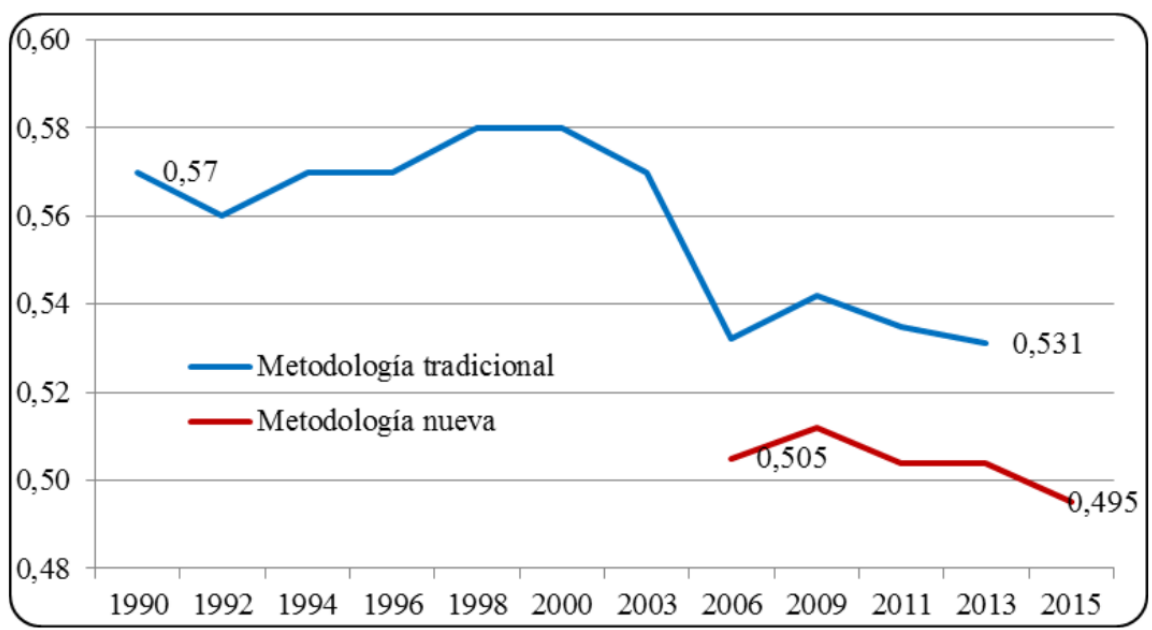

Fuente: Elaboración propia en base a Ministerio de Desarrollo Social (2015a, 2016b).

\section{TABLA 2}

INGRESO AUTONOMO MEDIO PER CAPITA Y DEL HOGAR, POR DECILES (CLP), CHILE 2015

\begin{tabular}{|c|c|c|c|c|}
\hline Decil & $\begin{array}{c}\text { Ingreso } \\
\text { autónomo } \\
\text { del hogar }\end{array}$ & $\begin{array}{c}\text { Veces } \\
\text { el primer decil }\end{array}$ & $\begin{array}{c}\text { Ingreso } \\
\text { per cápita } \\
\text { aut. del hogar }\end{array}$ & $\begin{array}{c}\text { Veces } \\
\text { el primer decil }\end{array}$ \\
\hline 1 & 105.179 & 1 & 27.550 & 1 \\
2 & 281.059 & 2,7 & 73.638 & 2,7 \\
3 & 375.933 & 3,6 & 102.405 & 3,7 \\
4 & 456.074 & 4,3 & 130.552 & 4,7 \\
5 & 553.829 & 5,3 & 161.758 & 5,9 \\
6 & 652.968 & 6,2 & 199.032 & 7,2 \\
7 & 776.195 & 7,4 & 249.767 & 9,1 \\
8 & 970.015 & 9,2 & 326.378 & 11,8 \\
9 & 1.281 .418 & 12,2 & 476.613 & 17,3 \\
10 & 2.865 .760 & 27,2 & 1.241 .962 & 45,1 \\
\hline
\end{tabular}

Fuente: Elaboración propia en base a Ministerio de Desarrollo Social (2016b). 
A través de los años, al igual que lo ilustrado por la evolución del Gini, la razón 10/10 muestra un aumento de las disparidades en el año 2009, donde el $10 \%$ más rico llega a distanciarse 38,8 veces del ingreso medio del $10 \%$ más pobre, para luego descender paulatinamente. El ratio fue de 27,6; 29,8; 29,1 y 27,2 para 2009, 2011, 2013 y 2015 respectivamente (calculado por Ministerio de Desarrollo Social [2016b], bajo la nueva metodología).

Del mismo modo, se calcula el ratio 10/10 para cada una de las regiones de Chile. En los informes del Ministerio de Desarrollo Social se suele calcular este ratio utilizando el promedio del ingreso autónomo total del hogar; otros estudios utilizan el ingreso autónomo de las personas, con el objeto de medir la desigualdad proveniente del mercado. Sin embargo, en este estudio se ha optado por utilizar el ingreso autónomo de los hogares, por ser la metodología que seguirá utilizando la CASEN, al considerarse más realista respecto de la situación social de las familias chilenas y por ser de gran utilidad para la aplicación de la política pública.

La Tabla 3 presenta los valores para el año 2015, exponiendo los montos del primer y último decil, así como el ratio 10/10, ordenado de forma descendente de

TABLA 3

DECILES E INDICE 10/10 REGIONES DE CHILE, AÑO 2015

\begin{tabular}{|l|c|c|c|}
\hline \multicolumn{1}{|c|}{ Región } & Primer decil & Ultimo decil & Indice 10/10 \\
\hline La Araucanía & 50.042 & 2.243 .071 & 44,8 \\
Los Ríos & 84.276 & 2.524 .631 & 30 \\
Los Lagos & 77.905 & 2.082 .140 & 26,7 \\
Biobío & 82.674 & 2.196 .896 & 26,6 \\
Maule & 98.152 & 2.108 .495 & 21,5 \\
Magallanes & 161.199 & 3.323 .768 & 20,6 \\
Aysén & 131.556 & 2.663 .647 & 20,2 \\
RM & 216.634 & 4.306 .086 & 19,9 \\
O’Higgins & 129.971 & 2.379 .143 & 18,3 \\
Coquimbo & 134.954 & 2.316 .379 & 17,2 \\
Tarapacá & 255.970 & 4.139 .002 & 16,2 \\
Valparaíso & 167.604 & 2.491 .731 & 14,9 \\
Atacama & 187.331 & 2.774 .205 & 14,8 \\
Antofagasta & 250.420 & 3.631 .412 & 14,5 \\
Arica y Parinacota & 184.834 & 2.234 .749 & 12,1 \\
\hline
\end{tabular}

Fuente: Elaboración propia en base a CASEN 2015. 
acuerdo a este último resultado. Bajo este concepto se marcan aún más las diferencias norte-sur, ya que las regiones más desiguales son La Araucanía, Los Ríos y Los Lagos (regiones del sur), mientras que las regiones mejor posicionadas son Arica y Parinacota, Antofagasta y Atacama (regiones del norte). Cabe mencionar que los altos resultados del ratio 10/10 de las regiones sureñas se deben principalmente a los bajos ingresos provenientes del primer decil. Mientras un hogar, dentro del 10\% más pobre, en la Araucanía puede recibir una media de 50.042 pesos chilenos, en la Región Metropolitana este monto se cuadruplica.

Al realizar el mismo ejercicio pero considerando el $20 \%$ de los hogares con mayores y menores ingresos, el ratio es claramente menor. Sin embargo, el escenario no cambia demasiado al jerarquizar el territorio, al menos no para las regiones mejor y peor catalogadas anteriormente. El Gráfico 7 demuestra nuevamente que La Araucanía y Los Ríos son los territorios más desiguales, seguida del Biobío y la Región Metropolitana, la cual sube al puesto número cuatro. Por su lado, con menor desigualdad destacan las regiones de Arica y Parinacota, Atacama y Antofagasta (las misma regiones destacadas en el ratio 10/10, pero en un orden diferente).

A nivel territorial, la Tabla 4 presenta el índice de Gini por regiones calculado por Ministerio de Desarrollo Social, ex-MIDEPLAN, utilizando la metodología tradicional (aplicado a 13 regiones), es decir, medido por personas y no por hogares. La información está ordenada de forma descendente de acuerdo a la mayor disminución de su nivel de desigualdad (1990-2011). Se observa que La Araucanía cada año encabeza la lista de las regiones más desiguales, a excepción del año 1990, donde ocupa el segundo lugar después de la región del Maule. En el año 2006, La Araucanía comparte el primer puesto con la Región Metropolitana. Cabe destacar, que lamentablemente, estas dos regiones son las que menos han disminuido las desigualdades de ingreso en el período 1990-2011, junto con la región de Aysén, la cual en el período de 21 años analizado, aumentó su desigualdad en casi un $2 \%$. Las regiones con menor desigualdad varían un poco cada año, sin embargo, algunas de ellas se repiten en el tiempo, como son algunas regiones del norte del país (Atacama y Antofagasta). La mayor disminución en el tiempo se percibe en la región del Maule (20\%), seguida de Coquimbo (16,4\%) y O'Higgins (15,7\%).

En este estudio, se calcula y compara el índice de Gini regional 2013 y 2015 (para las 15 regiones). Para ello se opta por utilizar la metodología recomendada por Medina (2001) y utilizada también, por el Ministerio de Desarrollo Social. En este caso particular se trabaja con datos agrupados para el cálculo del Gini. Se analizaron los ingresos per cápita autónomos (siguiendo la metodología tradicional) y los ingresos autónomos del hogar (siguiendo la metodología nueva) en base a los deciles autónomos per cápita regionales, aportados por las bases de datos CASEN 2013 y 2015. La fórmula utilizada es la siguiente: 


$$
G I N I=\frac{1}{2 n^{2} \bar{y}} * \sum_{j=1}^{n} \sum_{i=1}^{n}\left|y_{i}-y_{j}\right|
$$

Donde:

$n=$ número de grupos o deciles

$\bar{y}=$ ingreso promedio (por persona) en el conjunto de la población

$y_{s}=$ ingreso promedio (por persona) en el decil $\mathrm{s}$

\section{GRAFICO 7}

INDICE 20/20 REGIONES DE CHILE AÑO 2015

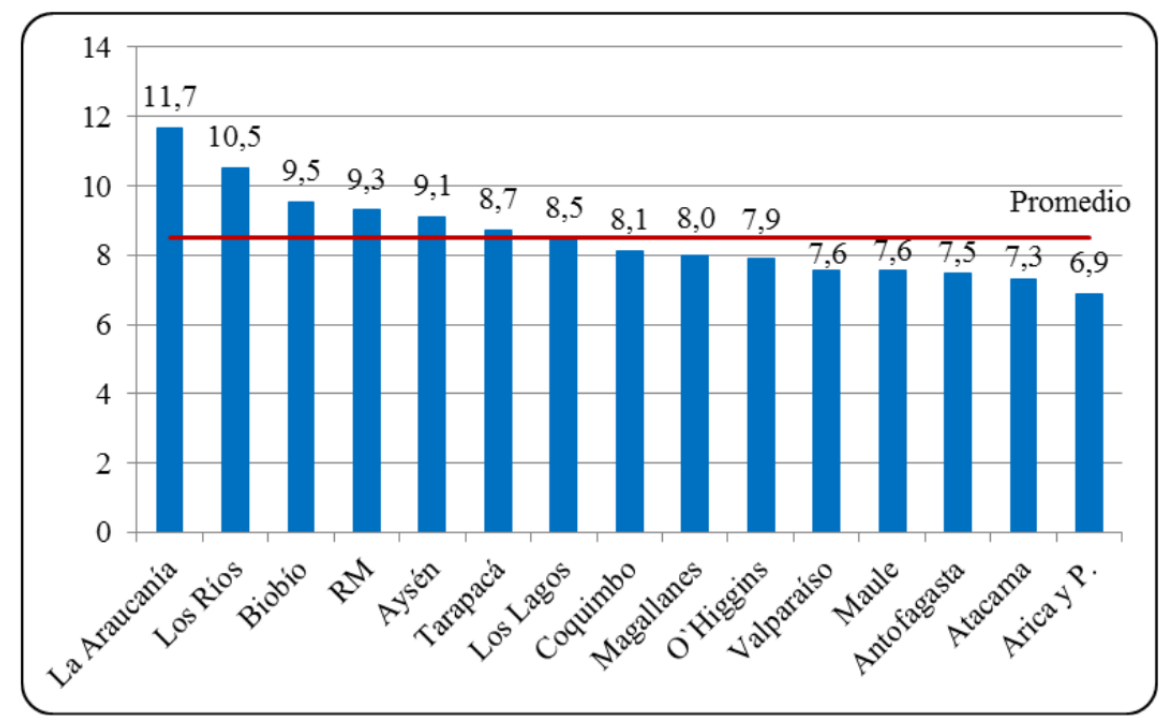

Fuente: Elaboración propia datos CASEN 2015. 


\section{TABLA 4}

INDICE DE GINI METODOLOGIA TRADICIONAL 1990-2011

\begin{tabular}{|l|c|c|c|c|c|c|}
\hline \multicolumn{1}{|c|}{ Región } & 1990 & 2000 & 2006 & 2009 & 2011 & $\begin{array}{c}\text { Variación } \\
1990-2011\end{array}$ \\
\hline Maule & 0,60 & 0,58 & 0,52 & 0,54 & 0,48 & $-20,00$ \\
Coquimbo & 0,55 & 0,55 & 0,51 & 0,48 & 0,46 & $-16,36$ \\
O’Higgins & 0,51 & 0,51 & 0,49 & 0,47 & 0,43 & $-15,69$ \\
Tarapacá & 0,55 & 0,52 & 0,47 & 0,44 & 0,48 & $-13,64$ \\
Atacama & 0,54 & 0,47 & 0,47 & 0,46 & 0,48 & $-11,11$ \\
Antofagasta & 0,52 & 0,52 & 0,46 & 0,42 & 0,47 & $-9,62$ \\
Los Lagos & 0,58 & 0,53 & 0,52 & 0,52 & 0,53 & $-8,62$ \\
Biobío & 0,58 & 0,60 & 0,53 & 0,51 & 0,54 & $-6,90$ \\
Valparaíso & 0,53 & 0,50 & 0,49 & 0,49 & 0,50 & $-5,66$ \\
Magallanes & 0,53 & 0,57 & 0,50 & 0,51 & 0,51 & $-3,77$ \\
La Araucanía & 0,59 & 0,61 & 0,55 & 0,58 & 0,57 & $-3,39$ \\
RM & 0,56 & 0,57 & 0,55 & 0,57 & 0,55 & $-1,79$ \\
Aysén & 0,52 & 0,53 & 0,46 & 0,57 & 0,53 & 1,92 \\
\hline
\end{tabular}

Fuente: Elaboración propia en base a Arredondo (nd); Ministerio de Desarrollo Social (2018).

Los resultados se exponen en la Tabla 5. Con la nueva metodología, se aprecia que en ambos años se repiten tanto las regiones más desiguales como aquellas con menor nivel de inequidad. Siguiendo la tendencia de los años anteriores, La Araucanía y la Región Metropolitana ocupan el primer y segundo lugar, con un índice de Gini de 0,444 y 0,434 respectivamente en 2015 y de 0,453 y 0,452 en 2013. Las regiones con menor desigualdad por ingreso en ambos períodos son Arica y Parinacota y Atacama (intercambiando de lugar entre un período y otro). Sin embargo, las regiones que han logrado reducir más sus niveles de desigualdad en el último período son las regiones de Los Lagos y Valparaíso, y en tercer lugar la Región Metropolitana, con un menor 4,01\%. Utilizando esta metodología de cálculo de datos agrupados, el índice de Gini para el país es de 0,445 en 2013 y 0,432 en 2015. Bajo este concepto, solo las dos regiones más desiguales estarían sobre el promedio nacional en ambos años. Al considerar los ingresos autónomos per cápita, observamos algunas diferencias, principalmente en la variación del Gini. Se aprecia que bajo esta metodología la disminución promedio es menor que bajo la nueva metodología, sin embargo, el comportamiento en ambos casos es similar. 


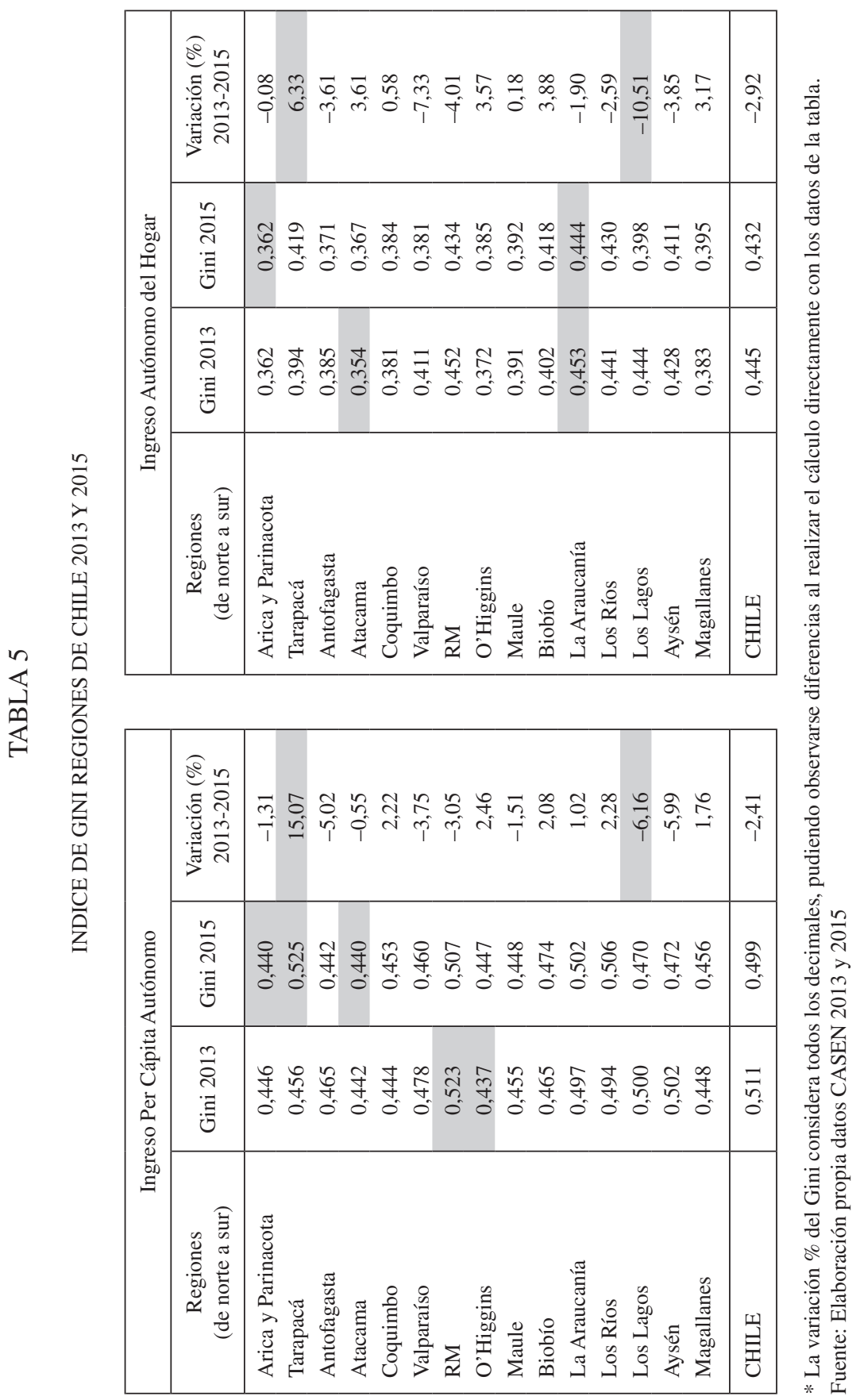


Mientras que a nivel nacional no hay un gran cambio en la desigualdad en el último período, Tarapacá incrementó su desigualdad en más de un $6 \%$, y por su parte Los Lagos la redujo en casi un $11 \%$ (metodología nueva). Estas diferencias son de gran relevancia para la política pública y de acuerdo con Contreras (1999), también para el proceso de descentralización. El autor argumenta que dentro de las posibles causas de las diferencias regionales en el Gini, está que las regiones crecen a ritmos distintos, además concentran sus actividades productivas en diferentes sectores de la economía. Las diferencias productivas tienen un efecto sobre los niveles de ingreso y su variación. Además, las regiones están expuestas a fluctuaciones económicas y naturales diferentes.

\section{INDICADORES DE DESARROLLO Y DESIGUALDAD SOCIAL}

La desigualdad de ingreso está estrechamente relacionada con la desigualdad social. Mayores ingresos son consecuentes con mejores oportunidades de acceso a un sistema educacional y de salud de calidad $^{14}$. Una persona de mayores ingresos irá a mejores escuelas, podrá asistir a clases particulares y extracurriculares, tendrá mejor asistencia dental, podrá ir a mejores centros médicos e incluso tomar mejores medicinas, que una persona pobre. Por tanto, peores condiciones sociales pueden aumentar las brechas entre ricos y pobres.

El desarrollo económico pone en contexto la calidad de vida de las personas. Ya se estudió uno de sus componentes, el ingreso, por tanto, en esta sección se estudiarán otros dos, la educación y la salud. En este apartado se examina el desarrollo económico del país, evaluado a través de índices de desarrollo humano (PNUD) y el índice de desarrollo regional (Vial, 2017) y a continuación se evalúa la desigualdad social en dos aspectos muy controversiales actualmente en el país, educación y salud. Ambos temas generan constantes polémicas, protestas y levantamientos por parte de la sociedad, la cual siente la desigualdad y busca que el Gobierno doble sus esfuerzos para satisfacer estas necesidades básicas, de una manera eficiente y que beneficie a toda la población y no solo a unos pocos. Los datos educacionales comparativos por país se obtienen de Barro y Lee (2010) y PNUD (2015). Los datos regionales, como años de escolaridad promedio y analfabetismo, se obtienen a partir de la Encuesta CASEN.

\subsection{Indices de Desarrollo}

El crecimiento económico es una condición necesaria para el desarrollo, sin embargo, no suficiente. En el libro de March (2013) se cita a Sunkel \& Paz (1986),

14 En Chile, el sistema público de educación, sobre todo educación básica y media, debe mejorar en calidad para ser competitivo con el sector privado. Por su parte, el sistema de salud presenta problemas, principalmente, por la falta de atención. 
quienes definen desarrollo como un proceso de cambio social, cuyo objetivo es igualar las oportunidades sociales, económicas y políticas, refiriéndose a un nivel nacional y a las sociedades que alcanzan mayores niveles de bienestar. En este mismo libro se hace referencia a Furtado (1983) quien considera que el desarrollo tiene tres dimensiones, “(1) la del incremento de la eficacia del sistema social de producción, (2) la de la satisfacción de las necesidades elementales de la población y (3) la de la consecución de objetivos oportuna a los que aspiran los grupos dominantes de una sociedad y que compiten en la utilización de recursos escasos" (pp. 20). Todaro y Smith (2012) por su lado, adopta la definición desarrollada por Amartya $\operatorname{Sen}^{15}$, donde se relacionan el proceso de desarrollo con la calidad de vida y con la capacidad de las personas de elevar su estatus, adicionando los conceptos de autoestima y libertad. En este último sentido, el Programa para las Naciones Unidas y el Desarrollo (PNUD) en 2016, hace referencia a las libertades humanas como "la libertad de desarrollar todo el potencial de cada vida humana -no solo el de unas pocas ni tampoco el de la mayoría, sino el de todas las vidas de cada rincón del planeta- ahora y en el futuro" (pp. 30).

Si bien el crecimiento es un fenómeno relativo al mercado y al aumento del PIB, como se ha mencionado, el desarrollo suma a esto el bienestar social, el cual se observa en la calidad de vida de la población, en el desarrollo de competitividad y capital humano, en la mayor calidad y acceso a educación y salud, entre otros factores. En esta oportunidad se estudiaran el índice de desarrollo humano (IDH) y el índice de desarrollo regional (IDERE).

El PNUD, cada año, desde 1990, presenta un informe sobre desarrollo humano, donde se analizan importantes temas, tendencias y políticas sobre desarrollo. No se puede negar que durante el último cuarto de siglo a nivel mundial ha habido un importante progreso en muchos ámbitos del desarrollo humano. La esperanza de vida es mayor; hay más personas que se escapan de la línea de la pobreza y la pobreza extrema; menos personas afectadas por la malnutrición, entre otros. Si bien es claro que estos avances han mejorado la calidad de vida de las personas, se debe asumir que no en la misma proporción e incluso ha dejado mucha gente marginada de este gran avance (PNUD, 2016). Es por lo anterior que el último informe del PNUD está dedicado a este tema "desarrollo humano para todos", considerando, igualmente, que en 2015 los dirigentes nacionales se comprometieron a "no dejar a nadie atrás", compromiso que dejaron plasmado en la Agenda 2030.

Considerando el IDH se realiza un ranking y se clasifica a los países como naciones con bajo, medio, alto o muy alto nivel de desarrollo humano. El IDH mide el promedio de los avances de un país en tres dimensiones básicas (con igual ponderación): una vida larga y saludable, medida por la esperanza de vida al nacer; la educación, medida por la tasa de alfabetización de adultos (con una ponderación de dos tercios) y la tasa bruta combinada de matriculación en escuelas primarias,

15 Puede ver Sen (2000), en El desarrollo como libertad. 
secundarias y terciarias (con una ponderación de un tercio) y un nivel de vida digno, medido por el PIB per cápita en términos de paridad del poder adquisitivo (PPA) en dólares estadounidenses (PNUD, 2017).

En el informe del PNUD (2016) ${ }^{16}$, se observa que Chile está dentro de los países con un nivel muy alto de desarrollo humano, ubicándose en el puesto número 38 , bajo Lituania y sobre Arabia Saudita, con una expectativa de vida promedio de 82 años, y una media de años de escolarización de 9,9 (de los 16,3 años esperados para el país). Dentro de esta categoría los únicos países latinoamericanos representados son Chile y Argentina en el puesto 45 (con valores de 0,847 y 0,827 respectivamente, siendo el promedio de América Latina y el Caribe de 0,751). Sin embargo, Chile retrocede 12 puestos si se considera la desigualdad ${ }^{17}$, quedando al nivel de países como Rumania, Kuwait o Rusia. Los primeros puestos son ocupados por Noruega, Australia y Suiza, mientras que en los peores lugares están la República Centroafricana, Nigeria y Chad, con niveles de desarrollo humano muy bajo.

A nivel de territorio chileno ${ }^{18}$, resulta interesante evaluar el IDH por regiones, lamentablemente, estos datos solo se encuentran disponibles para el año 2003 gracias al PNUD y Mideplan (2005). En la Tabla 6 se puede apreciar que para el año 2003 los IDH más bajos correspondían a las regiones del Maule y La Araucanía, con un valor de 0,675 y 0,679 respectivamente. Resultados que actualmente podríamos comparar con los obtenidos por Bolivia y el Salvador, países con un nivel de desarrollo humano medio. Por su parte, las regiones con mayor desarrollo humano, como era de esperar, fueron la Región Metropolitana y la región de Magallanes, con resultados comparables actualmente con México y Santa Lucía respectivamente, países con alto nivel de desarrollo humano. Respecto del año 1994 las regiones que mejoran son Magallanes, Aysén, Coquimbo y Antofagasta. Por su lado, mantienen su posición relativa, las regiones Metropolitana, de Los Lagos, de La Araucanía, del Biobío y del Maule. Mientras que bajan de posición relativa las regiones de Tarapacá (aunque continúa dentro de las con mayores logros de desarrollo humano), Atacama, O'Higgins y Valparaíso. Cabe mencionar que para 2003, solo cuatro regiones están sobre el promedio país.

Ante la escasez de datos actualizados del IDH por regiones, es que se recurre al índice de desarrollo regional (IDERE). Esta herramienta mide el desarrollo a nivel territorial desde un punto de vista multidimensional, se calcula a través de la media geométrica de índices normalizados que van de cero a uno, donde cero representa el nivel mínimo de desarrollo y uno el máximo (Vial, 2016). Las dimensiones

16 Reporte 2016 utiliza los datos del año 2015

17 El IDH-D considera las mismas tres dimensiones del IDH, pero considerando cómo se distribuyen los logros a lo largo de la población, penalizando el valor promedio para cada dimensión en función de lo desigual que sea. Si los países se encontraran en una situación ideal de perfecta igualdad, ambos indicadores coincidirían. Mientras mayor desigualdad, el IDH-D se ubicará más por debajo del IDH, siendo este el caso de Chile.

18 Tarapacá lo conformaban las actuales regiones de Arica y Parinacota y Tarapacá. La región de Los Lagos la conformaban las actuales regiones de Los Ríos y Los Lagos. 
consideradas por el IDERE son seis: educación, salud, bienestar económico, actividad económica, conectividad y seguridad. A partir del año 2017 se incorpora la variable medio ambiente y sustentabilidad ${ }^{19}$.

TABLA 6

VALOR IDH REGIONAL 1994-2003

\begin{tabular}{|l|c|c|c|c|c|}
\hline $\begin{array}{c}\text { Regiones (de } \\
\text { norte a sur) }\end{array}$ & IDH 1994 & IDH 2003 & $\begin{array}{c}\text { Ranking } \\
\text { regional } \\
\text { IDH 1994 }\end{array}$ & $\begin{array}{c}\text { Ranking } \\
\text { regional } \\
\text { IDH 2003 }\end{array}$ & $\begin{array}{c}\text { \% Reducción } \\
\text { distancia de la } \\
\text { meta ideal de } \\
\text { IDH 1994-2003 }\end{array}$ \\
\hline Tarapacá & 0,700 & 0,731 & 2 & 3 & 10,2 \\
Antofagasta & 0,666 & 0,729 & 5 & 4 & 18,9 \\
Atacama & 0,657 & 0,716 & 6 & 7 & 17,1 \\
Coquimbo & 0,646 & 0,717 & 7 & 6 & 20,2 \\
Valparaíso & 0,667 & 0,719 & 4 & 5 & 15,6 \\
RM & 0,700 & 0,760 & 1 & 1 & 19,8 \\
O'Higgins & 0,627 & 0,689 & 8 & 9 & 16,7 \\
Maule & 0,595 & 0,675 & 13 & 13 & 19,7 \\
Bío-Bío & 0,608 & 0,686 & 10 & 10 & 20 \\
Araucanía & 0,595 & 0,679 & 12 & 12 & 20,6 \\
Los Lagos & 0,598 & 0,681 & 11 & 11 & 20,7 \\
Aysén & 0,623 & 0,711 & 9 & 8 & 23,3 \\
Magallanes & 0,684 & 0,733 & 3 & 2 & 15,4 \\
\hline País & 0,659 & 0,725 & & & 19,3 \\
\hline
\end{tabular}

Fuente: Transcrita de PNUD y Mideplan (2005).

La Tabla 7 muestra, por una parte, la evolución de este índice considerando solo las seis dimensiones originales, lo que permite hacer una correcta comparación en el tiempo, y a continuación, expone los resultados 2017 al incorporar la variable de medio ambiente y sustentabilidad. Los resultados 2017 (seis dimensiones) muestran que las regiones con mayor IDERE son La Metropolitana y Magallanes, mientras que los peores resultados los presenta La Araucanía y el Maule, sin embargo, son de las regiones con mayor progreso durante el período evaluado. Al parecer se cumple el principio de convergencia, ya que algunas de las regiones con menor índice, son de las que más mejoran, mientras que las de mayor desarrollo tienen un progreso más lento (Metropolitana, Antofagasta, Valparaíso y Magallanes). La excepción a este

19 Para ver las variables que componen las distintas dimensiones, revisar el informe de Vial (2017). 
comportamiento ha sido Arica y Parinacota, que a pesar de su relativamente bajo IDERE, ha experimentado menor evolución en el período analizado.

La incorporación de la dimensión sustentabilidad y medio ambiente produjo que el IDERE disminuyera en todas las regiones (Tabla 7). La región de Magallanes fue la menos afectada tras la incorporación de la nueva dimensión, con un IDERE que solo descendió un 1,86\%. A diferencia de las regiones Metropolitana y Antofagasta, con descensos de $7,47 \%$ y 7,45\% respectivamente, que fueron las más afectadas. Pese a esta generalizada incidencia a la baja, la nueva dimensión no generó grandes cambios en las posiciones regionales del IDERE global. La dimensión sustentabilidad y medio ambiente es de gran relevancia para el crecimiento económico sostenible. Es lamentable que no se tengan datos históricos respecto a la inversión pública en el área medioambiental, sin embargo, contamos con los datos de los últimos años, los cuales develan que en 2015 la inversión ascendió a más de 27 mil millones de pesos. A nivel regional las regiones con mayor presupuesto medioambiental ejecutado fueron La Araucanía, Biobío y Aysén, tres regiones del sur del país.

\section{TABLA 7}

EVOLUCION IDERE 2010-2017

\begin{tabular}{|l|c|c|c|c|c|c|c|}
\hline $\begin{array}{c}\text { Regiones } \\
\text { (de norte a sur) }\end{array}$ & 2010 & 2016 & 2017 & Posición & $\begin{array}{c}\text { Evolución } \\
2010-2017\end{array}$ & $\begin{array}{c}2017 \\
\text { (con 7 } \\
\text { dimensiones) }\end{array}$ & Posición \\
\hline Arica y Parinacota & 0,437 & 0,509 & 0,498 & 11 & $13,96 \%$ & 0,470 & 12 \\
Tarapacá & 0,430 & 0,549 & 0,563 & 5 & $30,93 \%$ & 0,529 & 5 \\
Antofagasta & 0,485 & 0,583 & 0,591 & 3 & $21,86 \%$ & 0,547 & 4 \\
Atacama & 0,402 & 0,501 & 0,507 & 9 & $26,12 \%$ & 0,494 & 8 \\
Coquimbo & 0,395 & 0,491 & 0,504 & 10 & $27,59 \%$ & 0,482 & 10 \\
Valparaíso & 0,474 & 0,578 & 0,591 & 4 & $24,68 \%$ & 0,559 & 3 \\
RM & 0,550 & 0,627 & 0,629 & 1 & $14,36 \%$ & 0,582 & 1 \\
O’Higgins & 0,402 & 0,495 & 0,498 & 12 & $23,88 \%$ & 0,479 & 11 \\
Maule & 0,361 & 0,468 & 0,480 & 14 & $32,96 \%$ & 0,456 & 14 \\
Bío-Bío & 0,378 & 0,503 & 0,518 & 7 & $37,04 \%$ & 0,498 & 6 \\
Araucanía & 0,294 & 0,424 & 0,440 & 15 & $49,66 \%$ & 0,418 & 15 \\
Los Ríos & 0,371 & 0,504 & 0,525 & 6 & $41,51 \%$ & 0,497 & 7 \\
Los Lagos & 0,386 & 0,499 & 0,514 & 8 & $33,16 \%$ & 0,489 & 9 \\
Aysén & 0,388 & 0,475 & 0,488 & 13 & $25,77 \%$ & 0,465 & 13 \\
Magallanes & 0,477 & 0,582 & 0,592 & 2 & $24,11 \%$ & 0,581 & 2 \\
\hline
\end{tabular}

Fuente: Elaboración propia en base a Vial (2017). 
En su informe 2017, Vial concluye que las dimensiones que muestran mayores diferencias o brechas a nivel interregional son las de bienestar económico, conectividad y seguridad. Igualmente este informe divide a las regiones en tres niveles relativos de desarrollo, que son: nivel de desarrollo relativo mayor, intermedio y menor. De acuerdo con los últimos resultados, solamente la región de La Araucanía se encontraría en el nivel menor; nueve regiones en el nivel intermedio y cinco en el nivel mayor (Región Metropolitana, Magallanes, Valparaíso, Antofagasta y Tarapacá). Espacialmente esto se traduce en que la mayor concentración de desarrollo se da en el norte y centro del país, tendiendo a bajar a medida que se va hacia el sur de Chile (exceptuando Magallanes en el extremo sur).

\subsection{Desigualdad en Educación}

"En Chile, en el ámbito educacional, las oportunidades que se tienen en la infancia parecen tener profundos efectos, casi determinantes, sobre las oportunidades futuras de una misma persona" (Senado de la Nación, 2012, pp. 74). Esta frase refleja una sensible conclusión a la que llega el estudio Retrato de la desigualdad en Chile y es que, en todo nivel educativo, las desventajas de origen socioeconómico de los chilenos, marcan el acceso, la trayectoria y los resultados de su educación. Es decir, que el éxito o fracaso escolar, está fuertemente determinado por los orígenes de las personas, marcando una desigualdad desde los inicios. Como se mencionó al comienzo de la sección, familias más ricas tienen acceso a mejores colegios, actividades extracurriculares, posibilidad de aprender idiomas con profesores particulares, participar de más actividades deportivas e incluso ampliar redes, todo aquello que le abrirá puertas para un futuro de mayores ingresos. Por su parte, familias pobres muchas veces deben decidir a qué hijo educar profesionalmente. Por tanto, las condiciones iniciales cumplen un rol importante para marcar las desigualdades futuras. Mejorar sustancialmente la oportunidad educacional en todas las regiones juega un rol esencial para disminuir las brechas que separan a los chilenos.

Los años de escolaridad promedio en Chile han tenido una favorable evolución en el tiempo. Se observa en el Gráfico 8 que en 1955, en promedio, los chilenos no alcanzaban a completar la enseñanza primaria (8 años), llegando a este nivel solo a partir de 1990. En 2014 el promedio fue de 9,8 años, por debajo de la educación obligatoria en el país (12 años) y de los años de escolaridad esperada de acuerdo con el PNUD (15,2 años para 2014). El país busca facilitar cada vez más el acceso a estudios superiores, de hecho la política pública pretende que a través de becas y créditos la educación esté al alcance de la población económicamente más vulnerable. Sin embargo, aún queda trabajo por hacer en este ámbito tan importante para la equidad.

De acuerdo con la Encuesta CASEN, los años de escolaridad promedio en Chile son superiores a los presentados anteriormente por Barro y Lee y el PNUD. A pesar de estas diferencias, son muy informativos y nos permiten hacer comparaciones entre los distintos territorios del país y su evolución en el tiempo. Acorde con la CASEN, 
los años de escolaridad promedio del país son 11, con una leve diferencia por género, 11,2 años para los hombres y 10,9 en el caso de las mujeres. Esta diferencia, aunque pequeña, se ha mantenido en el tiempo y es el mismo comportamiento presentado por Barro y Lee (2010). Otros datos interesantes que nos entrega la CASEN en materia de educación, es la diferencia de escolaridad por lugar de nacimiento y pertenencia a una etnia. En Chile, en el año 2015, el promedio de escolaridad para una persona nacida en el país era de 11 años, y para un inmigrante (cuya madre residía en otro país al momento del nacimiento) de 12,5 años. Asimismo, hay una diferencia promedio de un año de escolaridad dependiendo de si pertenece o no a una etnia, siendo el promedio de 10,1 años para la población indígena y 11,1 para la no indígena.

\section{GRAFICO 8}

AÑOS DE ESCOLARIDAD PROMEDIO CHILE 1955-2014

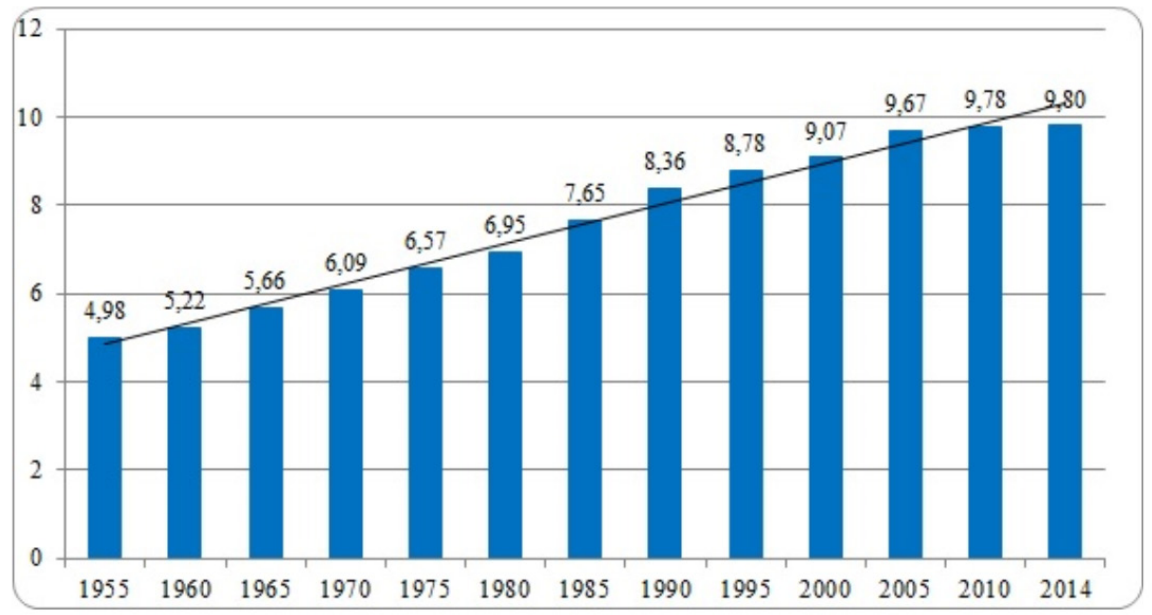

Elaboración propia datos Barro y Lee (2010), PNUD (2015).

A continuación se expone una comparación entre las regiones de Chile. La Tabla 8 presenta una variación en el tiempo de los años promedio de escolaridad. Se puede apreciar que cada año la región del Maule es aquella con el menor nivel de escolaridad, compartiendo en 2015 este último lugar con la región de Los Lagos. Ambas regiones superaron la media de pobreza del país, ya sea por ingresos o multidimensional. De hecho el Maule está en el grupo de regiones que menos logró disminuir la pobreza multidimensional en el período 2009-2015, como se vio en la Subsección 3.1. 
Por su lado, la Región Metropolitana, como no es de extrañar, ostenta los mejores resultados junto con regiones del norte como Antofagasta y Tarapacá. En 2015, seis de las 15 regiones superaban el promedio nacional calculado para ese año. Las regiones que más han mejorado su nivel de escolaridad promedio son Los Ríos, Aysén y Coquimbo, mientras que las regiones de menor avance han sido Atacama, la Región Metropolitana y Antofagasta. En general, las regiones con menores años de escolaridad al comienzo del período, son aquellas que han tendido a mejorar más este indicador.

\section{TABLA 8}

AÑOS DE ESCOLARIDAD PROMEDIO REGIONES DE CHILE

\begin{tabular}{|l|r|r|r|r|r|c|}
\hline $\begin{array}{c}\text { Regiones } \\
\text { (de norte a sur) }\end{array}$ & 2006 & 2009 & 2011 & 2013 & 2015 & $\begin{array}{c}\text { Variación } \\
2006-2015 \\
\text { (años) }\end{array}$ \\
\hline Arica y Parinacota & 10,5 & 11,1 & 10,9 & 11,0 & 11,4 & 0,90 \\
Tarapacá & 10,4 & 10,7 & 10,9 & 11,2 & 11,5 & 1,10 \\
Antofagasta & 10,7 & 10,6 & 11,2 & 11,5 & 11,5 & 0,80 \\
Atacama & 10,3 & 10,3 & 10,2 & 10,5 & 10,9 & 0,60 \\
Coquimbo & 9,6 & 9,7 & 10,1 & 10,5 & 10,7 & 1,10 \\
Valparaíso & 10,3 & 10,6 & 10,8 & 11,1 & 11,3 & 1,00 \\
RM & 10,8 & 11,2 & 11,2 & 11,4 & 11,6 & 0,80 \\
O’Higgins & 9,2 & 9,5 & 9,7 & 10,1 & 10,2 & 1,00 \\
Maule & 9,0 & 9,0 & 9,1 & 9,3 & 9,8 & 0,80 \\
Biobío & 9,7 & 9,9 & 9,8 & 10,2 & 10,6 & 0,90 \\
La Araucanía & 9,2 & 9,1 & 9,3 & 9,8 & 10,0 & 0,80 \\
Los Ríos & 9,0 & 9,3 & 9,7 & 9,8 & 10,2 & 1,20 \\
Los Lagos & 9,1 & 9,1 & 9,3 & 9,6 & 9,8 & 0,70 \\
Aysén & 9,4 & 9,5 & 9,9 & 9,9 & 10,6 & 1,20 \\
Magallanes & 10,3 & 10,2 & 10,8 & 11,1 & 11,2 & 0,90 \\
\hline Nacional & 10,1 & 10,4 & 10,5 & 10,8 & 11,0 & 0,90 \\
\hline
\end{tabular}

Elaboración propia datos Ministerio de Desarrollo Social (2016a).

En Chile, el analfabetismo es cada vez menor (ver Gráfico 9), pasando de un 5,2\% de la población de 15 años o más que no sabía leer ni escribir en 1990, a un 3,1\% en 2015. En 1990 un 14,2\% de la población rural era analfabeta y un 3,4\% de la población urbana; asimismo, el 5,4\% de las mujeres lo era, seguidas de cerca por un 4,9\% de los hombres. Para 2015 se observa un buen progreso en las personas del área rural, ya que un menor 7,8\% no sabe leer ni escribir, así como un 3\% de las personas de la ciudad; este año, hay un 3,3\% de mujeres analfabetas, seguidas muy de cerca por 
un 3\% de los hombres. A nivel regional, para el año 2015 se ven algunas diferencias importantes. Mientras en regiones como Tarapacá y Magallanes, el analfabetismo no alcanzó al 1\% de la población, en Los Ríos, La Araucanía y el Maule, superó el 4\%, develando una tarea pendiente en estas regiones, principalmente con su población rural. Para la mayoría de las regiones, hay un mayor porcentaje de mujeres analfabetas que de hombres, a excepción de las regiones de Tarapacá y O’Higgins.

\section{GRAFICO 9}

ANALFABETISMO POR REGIONES, 2015

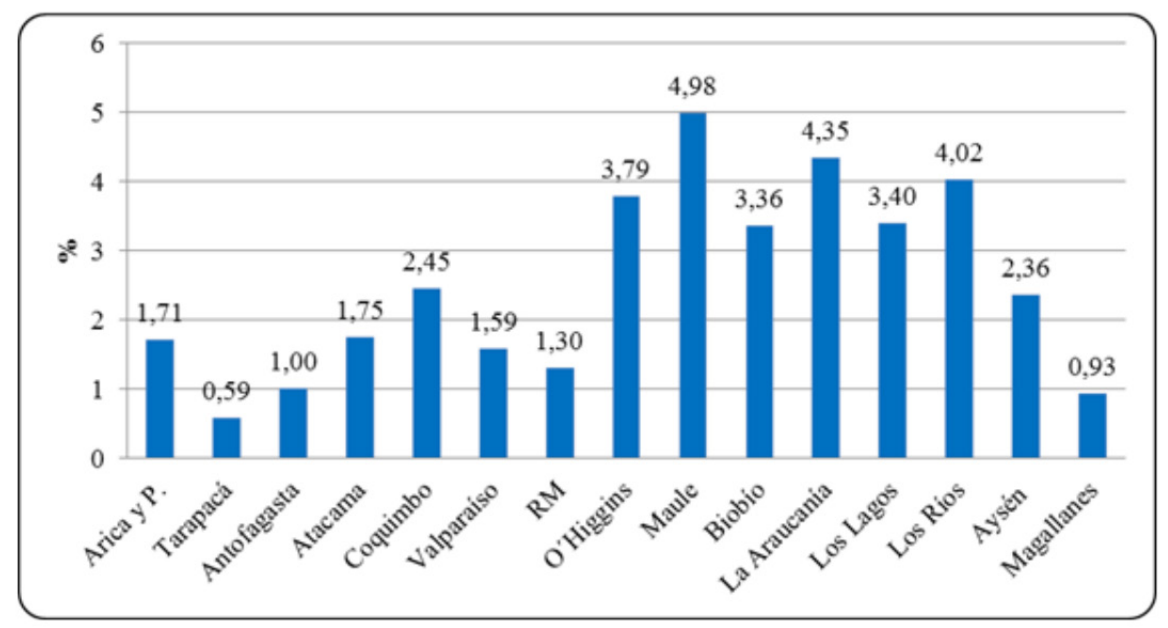

Fuente: Elaboración propia datos CASEN 2015.

\subsection{Desigualdad en Salud}

Para terminar la Sección 4, se revisará la dinámica de distintas variables del área de la salud en Chile y sus regiones. La pobreza y la mala salud son fenómenos interrelacionados, de acuerdo con Wagstaff (2002) la pobreza provoca una salud deficiente por distintas carencias a las que se ven enfrentadas las personas más pobres y, a su vez, existe pérdida de ingresos cuando se cuenta con mala salud. Es decir, esta mala salud se traduce en que los pobres sigan siendo pobres. Por consiguiente la salud deficiente fomenta las desigualdades del ingreso.

Chile ha presentado gran progreso en las últimas décadas en lo referente a indicadores de salud, con cifras comparables con países desarrollados en cuanto a mortalidad infantil y general. Sin embargo, igual como pasa con el crecimiento, estos 
logros no benefician a toda la población de la misma manera, existiendo diferencias a nivel regional. Este tipo de desigualdad suele crear malestar por parte de la ciudadanía y descontento hacia las autoridades públicas, a quienes se exige salud de calidad y para todos. Actualmente, en Chile el gasto total en salud representa el 7,2\% de su PIB, por debajo del promedio de los países miembros de la OCDE, 9,3\% (Instituto Nacional de Derechos Humanos, 2016), por lo que en materia de salud aún quedan muchos desafíos para el país y para la equidad en el territorio.

El país presenta un bajo índice de fertilidad de 1,8 nacimientos por mujer, cayendo por debajo de la tasa de reemplazo. Larrañaga y Herrera (2008) concluyen que en Chile, las tasas de fertilidad tienden a converger entre las mujeres de distintos estratos socioeconómicos, "mientras que a inicios de los sesenta la población de menor nivel socioeconómico tenía alrededor de 2,5 hijos más que la población de mayor nivel socioeconómico, a inicios de 2000 la brecha había disminuido a 1,0 hijo". Las familias más pobres habitualmente tienen más hijos, por tanto deben distribuir sus ingresos entre un mayor número de personas, incrementando los niveles de desigualdad. Por tanto, la convergencia señalada por los autores, tendería a reducir la brecha entre ricos y pobres. El Gráfico 10 da a conocer el comportamiento de las tasas brutas de natalidad, mortalidad y mortalidad infantil en el período 1980-2015. Se observa un comportamiento consecuente con las características de la segunda transición demográfica descrita por Vera (2012), donde tanto natalidad como mortalidad han disminuido en el largo plazo, sobre todo la mortalidad infantil. Si la tendencia continúa es posible que ambas tasas converjan. La natalidad es la variable que ha tenido una variación más significativa, disminuyendo en un $42 \%$ respecto a 1980.

Es interesante ver como a partir del año 2001 la tasa de mortalidad comienza nuevamente a ascender. De acuerdo con la teoría de la transición epidemiológica, propuesta y desarrollada por Abdel Omran desde 1971, el aumento de la TBM ocurriría porque si bien disminuyen los fallecimientos por factores exógenos, como enfermedades infecciosas y parasitarias, aumenta el peso relativo de muertes causadas por accidentes y factores endógenos, principalmente aquellos derivados de patologías degenerativas propias del envejecimiento, por ende se desplaza la mortalidad y morbilidad desde los jóvenes a los grupos de mayor edad (Frenk et al., 1991; Omran, 1998; Vera, 2012).

De acuerdo con los datos para 2015 del Banco Mundial, en Chile la esperanza de vida al nacer es de 79 años en promedio, siendo de 77 para los hombres y 81 en el caso de las mujeres, encontrándose sobre el promedio mundial (72 años, 70 para hombres y 74 para mujeres). A continuación se exponen las diferencias entre las regiones de Chile. Actualmente, en contraposición del año 1990, no existen grandes brechas entre la esperanza de vida de una u otra región, siendo esta de 2 años en 2015 y 5 años en 1990 (ver Gráfico 11). En 2015, Coquimbo y la Región Metropolitana lideran con casi 80 años en promedio, mientras que la menor esperanza de vida se observa en Antofagasta y la región de Los Lagos, con aproximadamente 78 años. Las mayores brechas se observan a nivel de género. En cada una de las regiones, en 2015, las diferencias van de los cuatro años (Arica y Parinacota) a los casi seis años de diferencia en Los Lagos, a favor de las mujeres. 


\section{GRAFICO 10}

TASA BRUTA DE NATALIDAD (TBN), TASA BRUTA DE MORTALIDAD (TBM) Y TASA BRUTA DE MORTALIDAD INFANTIL, CHILE 1990-2015

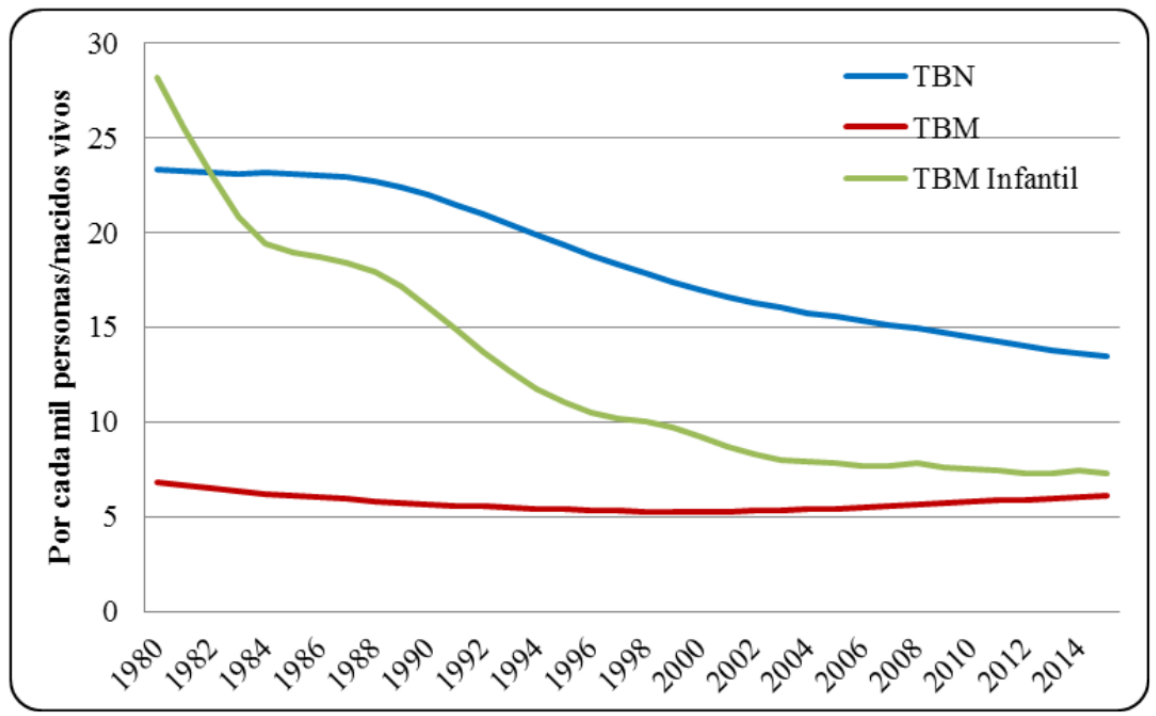

Fuente: Elaboración propia datos Banco Mundial.

A pesar de los buenos resultados expuestos y la pequeña diferencia promedio en la esperanza de vida, existen otras inequidades en el ámbito de la salud, como son el acceso al sistema de salud, los tiempos de espera hospitalarios y la cantidad de centros médicos o de profesionales de la salud disponibles por habitante, en cada una de las regiones.

De acuerdo con el informe 2016 del Instituto Nacional de los Derechos Humanos (INDH), existen inequidades en salud en regiones principalmente en dos áreas: la escasez de médicos especialistas y las brechas en infraestructura. Acorde con el Ministerio de Salud (2017), en 2015, a nivel nacional, existían más de 20.800 médicos especialistas, encontrándose la mayoría en la Región Metropolitana, Valparaíso y la región del Biobío, que concentran un $61 \%$ de los especialistas ${ }^{20}$ (ver Tabla 9). La mayor densidad de especialistas por cada 10.000 habitantes se observa en la Región Metropolitana y las zonas extremas de Magallanes y Aysén, mientras que las regiones

20 Lo no informado corresponde a especialistas que se desempeñan exclusivamente en el sector privado y son un $19 \%$ del total. 
del Maule, Atacama y Tarapacá tienen la menor densidad. Acorde con el informe del Instituto Nacional de Derechos Humanos (2016), el 62,1\% de los especialistas se desenvuelven en el sector privado, mientras que el $80 \%$ de la población chilena se atiende en el sector público.

\section{GRAFICO 11}

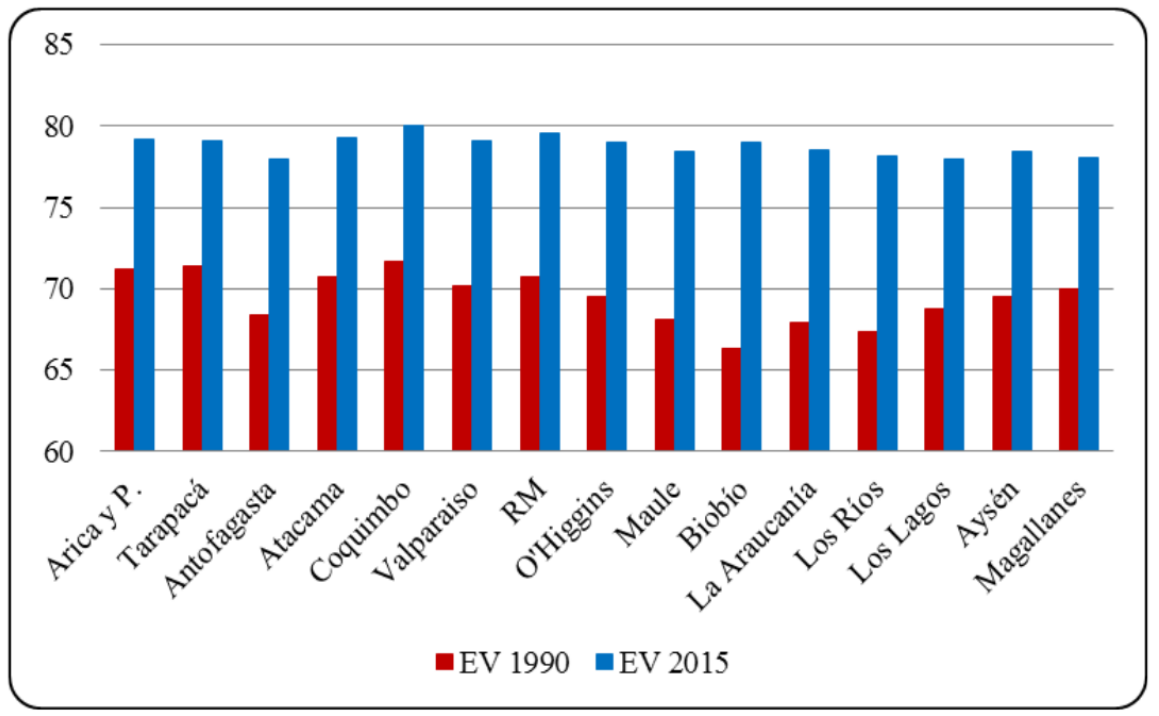

Fuente: Elaboración propia datos INE Chile.

Si bien, mejorar la salud es el principal objetivo de un servicio de salud, no es el único. Se debe, por un lado, alcanzar el mejor nivel posible, es decir un sistema de calidad, y por el otro lado, este sistema debe ser equitativo, "en este sentido, un sistema de salud es bueno si responde bien a lo que la gente espera de él; y es equitativo si responde igualmente bien a todos, sin discriminación"(OMS, 2000. Citado en INDH, 2016, pp. 194). 


\section{TABLA 9}

MEDICOS ESPECIALISTAS POR REGIONES DE CHILE 2015

\begin{tabular}{|l|c|c|c|}
\hline \multicolumn{1}{|c|}{$\begin{array}{c}\text { Región habitual } \\
\text { de trabajo }\end{array}$} & $\begin{array}{c}\text { Total Médicos } \\
\text { Especialistas } \\
\text { Certificados }\end{array}$ & $\begin{array}{c}\text { \% Médicos } \\
\text { Especialistas } \\
\text { por Región }\end{array}$ & $\begin{array}{c}\text { Especialistas } \\
\text { por cada } \\
10,000 \text { Hab. }\end{array}$ \\
\hline Arica y Parinacota & 156 & 0,75 & 6,52 \\
Tarapacá & 160 & 0,77 & 4,75 \\
Antofagasta & 393 & 1,88 & 6,31 \\
Atacama & 144 & 0,69 & 4,61 \\
Coquimbo & 419 & 2,01 & 5,43 \\
Valparaíso & 1.383 & 6,62 & 7,57 \\
RM & 9.874 & 47,29 & 13,50 \\
O'Higgins & 493 & 2,36 & 5,37 \\
Maule & 177 & 2,28 & 4,57 \\
Biobío & 1.460 & 6,99 & 6,91 \\
La Araucanía & 786 & 3,76 & 7,94 \\
Los Ríos & 336 & 1,61 & 8,31 \\
Los Lagos & 643 & 3,08 & 7,64 \\
Aysén & 96 & 0,46 & 8,86 \\
Magallanes & 170 & 0,81 & 10,32 \\
No informado & 3.889 & 18,63 & \\
\hline Total Nacional & $\mathbf{2 0 . 8 7 9}$ & $\mathbf{1 0 0}$ & $\mathbf{1 1 , 5 9}$ \\
\hline
\end{tabular}

Fuente: Elaboración propia datos Ministerio de Salud (2017).

\section{CONVERGENCIA DEL PIB PER CÁPITA Y DE LA DESIGUALDAD DEL INGRESO}

Con los datos regionales obtenidos, se realiza un análisis de convergencia para comparar si los territorios más pobres, crecen más rápido que los más ricos. De acuerdo con Barro y Sala-i-Martin (2009), una de las formas de encontrar la velocidad de convergencia $\beta$, es trabajar con un conjunto de datos de territorios que converjan hacia un estado estacionario similar, lo cual es posible encontrar en datos regionales. Lo anterior, debido a que las regiones de un mismo país suelen tener acceso a similares tecnologías, una cultura similar, gustos y preferencias parecidos y además, destacan los autores, tienen un mismo Gobierno Central, compartiendo leyes e instituciones. A pesar que es claro que las regiones tienen sus diferencias entre sí, estas son menores a las que se encontrarían al comparar distintos países. "Esta relativa homogeneidad significa que es más probable que exista convergencia absoluta entre regiones de un mismo país que entre países" (Barro y Sala-i-Martin, 2009, pp. 468).

A través de los datos analizados en este acápite se puede demostrar que existe convergencia entre las regiones de Chile en términos del PIB per cápita. Se evaluó en 
el largo plazo (1990-2016, con 13 regiones) y en el mediano plazo (2009-2016, con 15 regiones). Existe una correlación negativa entre el PIB per cápita inicial y la tasa de crecimiento anual promedio del PIB per cápita. El $\beta$ es negativo y significativo en ambos períodos (coeficiente $\beta$ de $-0,940$ y $-2,234$ respectivamente). Acorde a lo anterior, se concluye que las regiones más pobres inicialmente, tienden a crecer más rápido que las regiones inicialmente más ricas (Gráfico 12, a y b). Tendencia que se veía reflejada en las comparaciones regionales de los capítulos anteriores. Se observa mayor nivel de ajuste en el período 2009-2016.

Asimismo, se aplica la misma metodología, pero para estudiar la convergencia de la desigualdad del ingreso regional. Se analiza el índice de Gini al inicio del período frente a la variación promedio anual del Gini. Se observa una pendiente negativa en ambos períodos, demostrando que las regiones que son más desiguales al comienzo, han sido capaces de reducir en mayor cuantía sus niveles de desigualdad (Gráfico 13, a y b). En ambos períodos de tiempo el $\beta$ es negativo y significativo al 95 y $99 \%$ respectivamente (siendo los $\beta-4,256$ y $-24,851$ ). En el mediano plazo, se ve un mayor nivel de ajuste, con un $\mathrm{R}^{2}$ de 0,647 . La región de La Araucanía, siendo la más desigual en el año 2009, no es la que más ha reducido sus niveles de desigualdad, pero se encuentra dentro de las tres mejores. Las tres regiones del norte, Tarapacá, Antofagasta y Atacama, efectivamente fueron las regiones que iniciaron con los índices de Gini más bajos, logrando menores reducciones de desigualdad en el tiempo.

\section{GRAFICO 12}

ANALISIS DE CONVERGENCIA (ABSOLUTA)

EN RENTA PER CAPITA

(a)

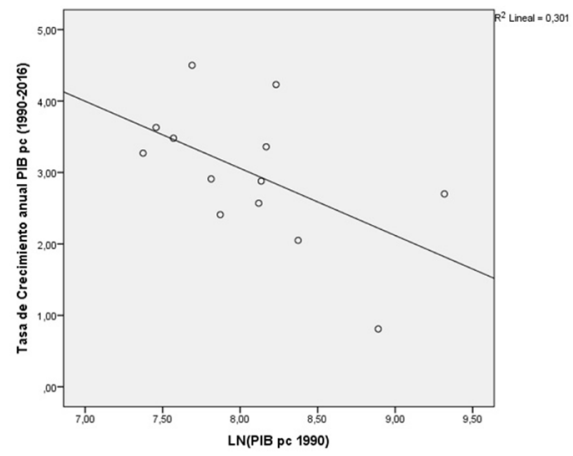

(b)

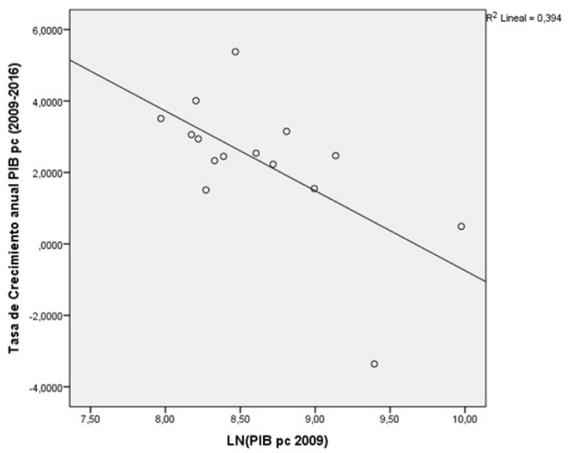

Fuente: Elaboración propia. 


\section{GRAFICO 13}

ANÁLISIS DE CONVERGENCIA (ABSOLUTA) DE LA DESIGUALDAD

(a)

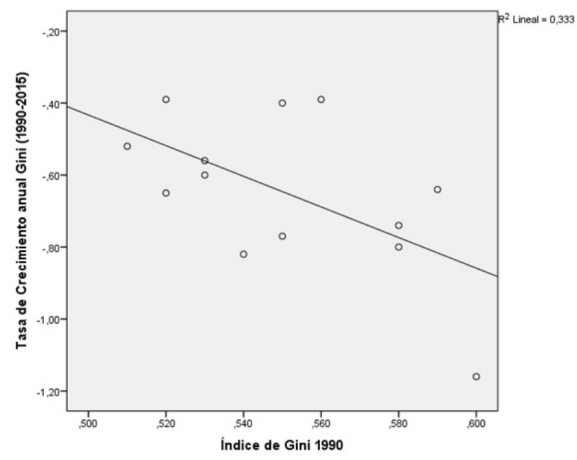

(b)

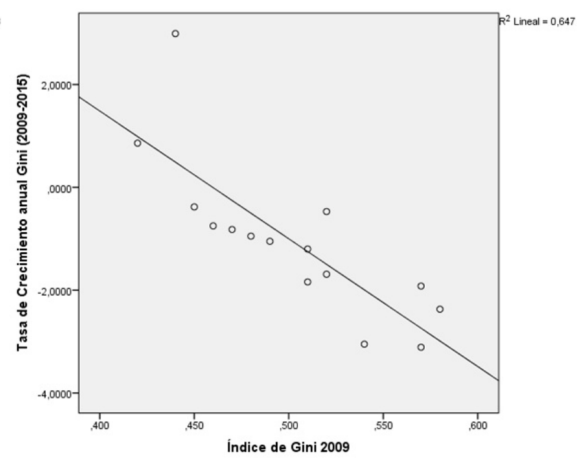

Fuente: Elaboración propia.

Este apartado concluye que, para los períodos estudiados, los territorios en peores condiciones iniciales (ya sea por ingreso o por desigualdad del ingreso), tienden a mejorar su condición respecto de los territorios más aventajados inicialmente. Aunque era de esperar que las regiones más pobres sean las que más rápido crezcan, no es obvio el comportamiento de la desigualdad. Si bien el índice de Gini converge, este sigue siendo alto y persistente.

Dicho lo anterior, es posible que las políticas redistributivas aún no sean desarrolladas con la eficacia que el país necesita para reducir más rápido las brechas económicas que le aquejan. De acuerdo con Donoso (2014), los gobiernos de la Concertación, principalmente desde la vuelta a la democracia, han tomado la inequidad como "espíritu de lucha", por lo que se han implementado una serie de políticas redistributivas (tales como la reforma educativa y tributaria), sin embargo, acorde con el autor, los gobiernos han fallado en este objetivo. Además de los indicadores de desigualdad conocidos, recalca que la sociedad, actualmente, valida más la desigualdad que hace 30 años. Asimismo, la percepción de este autor es que las políticas redistributivas, más que abogar por una verdadera equidad, buscan beneficios adicionales, como la perpetuación del poder político-económico.

Por su lado, Sunkel e Infante (2009), atribuyen la persistente desigualdad a la permanencia de la asimetría y falta de vínculos estrechos entre los diferentes estratos de la economía. Enfatizan en que los sectores más dinámicos y productivos de la economía, que se concentran en pocas empresas y generan una baja proporción del empleo, coexisten con sectores más atrasados tecnológicamente, con menos acceso a 
capital, mayor endeudamiento y menor productividad, donde se encuentra la mayoría de las empresas y que son responsables de más del $80 \%$ del empleo privado. Otros determinantes de la resistente desigualdad son "la concentración de la propiedad y el poder económico, el acceso diferenciado según los ingresos a la educación de calidad, la diferencia de remuneraciones en los empleos, la debilidad de los sindicatos, entre otros factores..." (Sunkel e Infante, 2009, pp. 89).

Dado que las cifras iniciales de desigualdad en Chile son muy altas, los avances han sido lentos y se necesitaría de un mayor esfuerzo por parte del Gobierno para someter todas aquellas fuerzas que amplifican las desigualdades.

\section{CONCLUSIONES}

La construcción de una amplia base de datos regional permitió dar una mirada a la dinámica de la desigualdad en Chile, retratando las diferencias regionales. En Chile existen grandes desigualdades entre sus regiones a nivel social y económico. La tabla del Anexo D resume los resultados de los indicadores evaluados en este trabajo, revelando la vulnerable posición de la región de La Araucanía, la peor catalogada en cada uno de los indicadores económicos y algunos sociales. A nivel de desarrollo económico, la Región Metropolitana logra los mejores resultados, mientras las regiones del sur del país, los peores, destacando nuevamente La Araucanía, única región catalogada con un bajo nivel de desarrollo, de acuerdo al IDERE.

Es lamentable ver que a nivel de desigualdad del ingreso, medido a través del índice de Gini, índice 10/10 o índice 20/20, las regiones del sur suelen encontrarse en una posición desfavorecida versus las regiones nortinas. Por su parte, la Región Metropolitana, tiene el segundo mayor índice de Gini del país, posición casi invariante a lo largo del período estudiado. Se observa que las regiones más desiguales, son igualmente aquellas con menor PIB per cápita y menores índices de desarrollo.

Es evidente que el PIB per cápita chileno ha crecido a nivel agregado y nacional. Se comprobó la existencia de convergencia absoluta en términos de PIB per cápita y desigualdad del ingreso. Las regiones más pobres tienden a crecer más, en promedio, que aquellas más ricas. De igual forma, las regiones con mayor desigualdad inicial, son las que tienden a reducir más rápido este indicador.

A pesar de la convergencia, la desigualdad de la renta es alta y persistente en el tiempo. Para reducir la resistente desigualdad, son necesarias más políticas distributivas, junto con las redistributivas, que se preocupen a priori de la generación del ingreso. Las políticas distributivas, en el largo plazo, permitirán reducir el gasto social enfocado en los más pobres, quienes con mayores oportunidades serán capaces, por sí mismos, de guiar su desarrollo.

Dado que las cifras iniciales de desigualdad en Chile son muy altas, los avances han sido lentos y se necesitaría de un mayor esfuerzo por parte del Gobierno para someter todas aquellas fuerzas que tiendan a aumentar las disparidades. Estos resultados 
motivaron el trabajo Develando los determinantes de la desigualdad del ingreso en Chile: Estudio empírico regional el que analizó, a través de un estudio econométrico, los principales factores que determinan la desigualdad del ingreso en Chile.

\section{REFERENCIAS}

AGOSTINI, C. y P. BROWN (2007). "Geographic income inequality in Chile", Revista de Análisis Económico 22 (1), pp. 3-33.

AGOSTINI, C. y P. BROWN (2007). "Inequality at low levels of aggregation in Chile", Review of Development Economics 14 (2), pp. 213-226.

ARREDONDO, J. (N.D.). "Revisión descriptiva de la desigualdad en el ingreso de las regiones de Chile". 10 de Diciembre de 2017, <http://www.ministeriodesarrollosocial.gob.cl/btca/txtcompleto/mideplan/ ser.estsoc-revis.desigualden-ing.dereg.pdf>.

BANCO CENTRAL DE CHILE (2018). Banco Central de Chile, Información Estadística. 06 de Febrero de 2018, <https://si3.bcentral.cl/Siete/secure/cuadros/home.aspx>.

BANCO MUNDIAL (2017). Banco Mundial, El Banco Mundial en Chile. 12 de Noviembre de 2017, $<$ http://www.bancomundial.org/es/country/chile/overview\#3>.

BANCO MUNDIAL (2018). Banco Mundial, Datos. 12 de Noviembre de 2017, <https://datos.bancomundial. org/>.

BARRO, R. y J. LEE (2010). Full Dataset: Education attainment for population aged 25 and over. 01 de Febrero de 2018, <http://www.barrolee.com/data/yrsch2.htm>.

BARRO, R. y X. SALA-I-MARTIN (2009). Crecimiento Económico, Reverté, Barcelona.

CONTRERAS, D. (1999). "Distribución del ingreso en Chile. Nueve hechos y algunos mitos", Perspectivas 2(2), pp. 311-332.

CORREA, V.; A. ESCANDON; R. LUENGO y J. VENEGAS (2002). "Empalme PIB: series anuales y trimestrales 1986-1995, base 1996. Documento metodológico", Banco Central de Chile, Working Paper (179).

DONOSO, J. (2014). “Políticas redistributivas en Chile. ¿Altruismo fuerte o altruismo débil?”, Estudios Nueva Economía 3 (1), pp. 5-7.

FONDO MONETARIO INTERNACIONAL (2017). Fondo Monetario Internacional, Perspectivas de la economía mundial. 21 de Diciembre de 2017, <http://www.imf.org/external/spanish/>.

FRENK, J.; T. FREJKA; J. L. BOBADILLA; C. STERN; R. LOZANO; J. SEPULVEDA y M. JOSE (1991). "La transición Epidemiológica en América Latina", Bol Oficina Sanit Panam 111, pp. 485-496.

GASPARINI, L.; M. CICOWIEZ y W. SOSA (2014). "Pobreza y desigualdad en América Latina: conceptos, herramientas y aplicaciones", Documentos de Trabajo del CEDLAS (171).

INSTITUTO NACIONAL DE DERECHOS HUMANOS (2016). "Situación de los Derechos Humanos en Chile, Informe Anual 2016”, INDH. 06 de Febrero de 2018, <https://www.indh.cl/bb/wp-content/ uploads/2017/01/Informe-Anual-INDH-2016.pdf>.

INSTITUTO NACIONAL DE ESTADISTICAS CHILE (2017). INE, Estadísticas Demográficas y Vitales. 06 de Febrero de 2019, <http://www.ine.cl/estadisticas/demograficas-y-vitales>.

KEELEY, B. (2015). Income Inequality: The Gap between Rich and Poor, OECD Insights, OECD Publishing, Paris. <http://dx.doi.org/10.1787/9789264246010-en>

KUZNETS, S. (1955). Economic growth and income inequality. The American economic review, 45(1): 1- 28.

LARRAÑAGA, O. y R. HERRERA (2008). "Los recientes cambios en la desigualdad y la pobreza en Chile", Estudios públicos (109), pp. 149-186.

MAC-CLURE, O. y R. CALVO (2013). "Desigualdades sociales y tipos de territorios en Chile", Polis. Revista Latinoamericana 12 (34), pp. 467-490.

MACIAS, A. (2014). "Crecimiento, desigualdad y pobreza: Estado de la cuestión”, Revista de economía institucional 16 (31), pp. 101-126. 
MARCH, J. M. (2013). Desarrollo económico: estilos y políticas, Tirant Lo Blanch, Valencia.

MEDINA, F. (2001). Consideraciones sobre el índice de Gini para medir la concentración del ingreso, CEPAL, Santiago de Chile.

MERCHAND, M. A. (2009). "Reflexiones en torno a la nueva geografía económica en la perspectiva de Paul Krugman y la localización de la actividad económica", Breves Contribuciones del IEG-Instituto de Estudios Geográficos "Dr. Guillermo Rohmender"- (21), pp. 206-223.

MIERES, M. (2015). Descentralización como herramienta de desarrollo económico en la región de La Araucanía, Chile (Tesis de Máster), Universidad de Valladolid, Valladolid, España.

MINISTERIO DE DESARROLLO SOCIAL (2015a). "Evolución y distribución del ingreso de los hogares (2006-2013)", Observatorio Social. 12 de Diciembre de 2017, <http://observatorio. ministeriodesarrollosocial.gob.cl/documentos/Casen2013_Evolucion_Distibucion_Ingresos.pdf>.

MINISTERIO DE DESARROLLO SOCIAL (2015b). "Informe de desarrollo social 2015", Observatorio Social. 12 de Diciembre de 2017, <www.ministeriodesarrollosocial.gob.cl/pdf/upload/IDS INAL FCM 3.pdf>.

MINISTERIO DE DESARROLLO SOCIAL (2015c). "Situación de pobreza en Chile", Observatorio Social. 20 de Diciembre de 2017, <http://observatorio.ministeriodesarrollosocial.gob.cl/casen_multidimensional/ casen/docs/CASEN_2015_Situacion_Pobreza.pdf>.

MINISTERIO DE DESARROLLO SOCIAL (2016a). "Educación, síntesis de resultados", Observatorio Social. 20 de Diciembre de 2017, <http://observatorio.ministeriodesarrollosocial.gob.cl/casen multidimensional/casen/docs/CASEN_2015_Resultados_educacion.pdf>.

MINISTERIO DE DESARROLLO SOCIAL (2016b). "Evolución y distribución del ingreso de los hogares". Observatorio Social. 12 de Diciembre de 2017, <http://observatorio.ministeriodesarrollosocial.gob. cl/casen_multidimensional/casen/docs/CASEN_2015_Ingresos_de_los_hogares.pdf>.

MINISTERIO DE DESARROLLO SOCIAL (2018a). Observatorio Social, Encuesta CASEN. 10 de Noviembre de 2017, <http://observatorio.ministeriodesarrollosocial.gob.cl/index.php>.

MINISTERIO DE DESARROLLO SOCIAL (2018b). Observatorio Social, Archivo Histórico CASEN. 10 de Noviembre de 2017, <http://observatorio.ministeriodesarrollosocial.gob.cl/casen/casen_obj.php>.

MINISTERIO DE SALUD (2017). "Informe sobre brechas de personal de salud por servicio de salud", Ministerio de Salud. 08 de Abril de 2018, <http://www.minsal.cl/wpcontent/uploads/2015/08/Infor meBrechasRHSenSectorP\%C3\%BAblico_Abril2017.pdf>.

MONTECINOS, E. (2005). "Antecedentes sobre la relación histórica centralismo y descentralización en Chile", Revista Venezolana de Gerencia 10 (31), pp. 433-462.

OCDE (2012). "OECD Economic Surveys: Chile 2012", OECD-iLibrary. 25 de Enero de 2018, <http:// dx.doi.org/10.1787/eco_surveys-chl-2012-en>.

OCDE (2013). "OECD Economic Surveys: Chile 2013”, OECD-iLibrary. 25 de Enero de 2018, <http:// dx.doi.org/10.1787/eco_surveys-chl-2013-en>.

OCDE (2015). "OECD Economic Surveys: Chile 2015", OECD-iLibrary. 26 de Enero de 2018. <http:// dx.doi.org/10.1787/eco_surveys-chl-2015-en>.

OCDE (2018). OCDE Estadísticas. 01 de Enero de 2018, <https://www.oecd.org/centrodemexico/estadisticas/>.

OCDE y CEPAL (2016). "Evaluaciones del desempeño ambiental Chile 2016", Repositorio Digital, CEPAL. 31 de Enero de 2018, <http://repositorio.cepal.org/handle/11362/40308>.

OMRAN, A. R. (1998). "The epidemiologic transition theory revisited thirty years later", World Health Statistics Quarterly 51 (2-4), pp. 99-119.

PALMA, E. (2009). "El desafío de la descentralización: la especificidad y las exigencias del caso chileno”, en Von Baer, H. (Ed.), Pensando Chile desde sus regiones, Universidad de la Frontera, Temuco, pp. 47-60.

PNUD (2015). "Informe sobre desarrollo humano 2015", PNUD. 12 de Febrero de 2018, <http://hdr.undp. org/sites/default/files/hdr_2015_report_sp.pdf>.

PNUD (2016). "Informe sobre desarrollo humano 2016", PNUD. 12 de Febrero de 2018, <http://hdr.undp. org/en/2016-report/download>.

PNUD (2017). Human development reports, United Nations development programme. 26 de Diciembre de $2017,<$ http://hdr.undp.org/en/home $>$. 
PNUD Y MIDEPLAN (2005). Las trayectorias del desarrollo humano en las comunas de Chile, 19942003, PNUD, Santiago de Chile.

RAMIREZ, E.; A. TARTAKOWSKY y F. MODREGO (2009). "Importancia de la desigualdad geográfica en Chile", Programa Dinámicas Territoriales Rurales (RIMISP), Documento de Trabajo $\mathrm{N}^{\circ} 30$.

SENADO DE LA NACION (2012). "Retrato de la desigualdad en Chile", Biblioteca del Congreso Nacional. 28 de Septiembre de 2017, <https://www.bcn.cl/obtienearchivo?id=documentos/10221.1/29929/1/ PDF_librodesigualdad_ultima_version.pdf $>$.

SOTO, R. y A. TORCHE (2004). "Spatial inequality, migration and economic growth in Chile", Cuadernos de economía 41 (124), pp. 401-424.

SUMMERS, R. y A. HESTON (2018). The Summer and Heston data set (Penn World Table version 9.0). 28 de Noviembre de 2017, <https://www.rug.nl/ggdc/productivity/pwt/pwt-releases/pwt9.0>.

SUNKEL, O. y B. INFANTE (Eds.) (2009). Hacia un desarrollo inclusivo: el caso de Chile, CEPAL, Fundación Chile XXI, OIT, Santiago de Chile.

TODARO, M. y S. SMITH (2012). Economic Development (11 a edición), Addison-Wesley, Boston.

VERA, X. (2012). “¿Hacia o en la segunda transición demográfica?: los cambios poblacionales de Chile desde un enfoque de género", Anales de la Universidad de Chile (3), pp. 105-125.

VIAL, C. (2016). "Indice de Desarrollo Regional IDERE 2016", IDERE. 08 de Enero de 2018, <http:// www.idere.cl/wpcontent/uploads/2016/10/IDERE2016digital.pdf>.

VIAL, C. (2017). "Indice de Desarrollo Regional IDERE 2017", IDERE. 08 de Enero de 2018, <http:// www.idere.cl/wp-content/uploads/2017/08/IDERE2017.pdf>.

WAGSTAFF, A. (2002). "Pobreza y desigualdades en el sector de la salud", Revista panamericana de salud pública 11 (5), pp. 316-326. 


\section{ANEXOS}

\section{A. Aporte al PIB regional por actividad económica (\%) 2016}

\begin{tabular}{|l|c|c|c|c|c|}
\hline Región/ Sector(\%) & $\begin{array}{c}\text { Agropecuario- } \\
\text { silvícola y } \\
\text { pesca }\end{array}$ & Minería & $\begin{array}{c}\text { Sector } \\
\text { primario } \\
(1+2)\end{array}$ & $\begin{array}{c}\text { Sector } \\
\text { Secundario }\end{array}$ & $\begin{array}{c}\text { Sector } \\
\text { Terciario }\end{array}$ \\
\hline Arica y Parinacota & 6,77 & 2,97 & 9,74 & 16,60 & 73,66 \\
Tarapacá & 0,52 & 38,01 & 38,53 & 13,00 & 48,47 \\
Antofagasta & 0,10 & 52,57 & 52,67 & 20,41 & 26,92 \\
Atacama & 2,33 & 41,16 & 43,48 & 20,52 & 36,00 \\
Coquimbo & 7,33 & 27,60 & 34,93 & 13,21 & 51,86 \\
Valparaíso & 4,18 & 8,67 & 12,85 & 28,00 & 59,15 \\
RM & 0,85 & 1,55 & 2,40 & 17,60 & 79,99 \\
O’Higgins & 12,95 & 23,11 & 36,05 & 22,07 & 41,88 \\
Maule & 13,53 & 1,73 & 15,26 & 28,35 & 56,40 \\
Biobío & 6,95 & 0,02 & 6,97 & 34,83 & 58,20 \\
La Araucanía & 10,19 & 0,00 & 10,19 & 21,61 & 68,20 \\
Los Ríos & 11,90 & 0,00 & 11,90 & 29,86 & 58,24 \\
Los Lagos & 11,95 & 0,00 & 11,95 & 27,64 & 60,41 \\
Aysén & 24,26 & 1,63 & 25,89 & 12,74 & 61,37 \\
Magallanes & 3,73 & 7,01 & 10,74 & 28,21 & 61,05 \\
\hline
\end{tabular}

Fuente: Elaboración propia en base a datos BC.

\section{B. Variación del PIB total por regiones de Chile y su aporte al PIB nacional}

\begin{tabular}{|l|r|r|r|r|}
\hline \multirow{2}{*}{ Región } & \multicolumn{3}{|c|}{ Variación \% anual del PIB } & \multirow{2}{*}{$\%$ del PIB } \\
\cline { 2 - 4 } & 2014 & 2015 & 2016 & nacional 2016 \\
\hline Arica y Parinacota & 1,73 & 3,69 & 2,31 & 0,72 \\
Tarapacá & 2,65 & $-0,57$ & 0,89 & 2,27 \\
Antofagasta & 4,59 & 0,79 & $-2,92$ & 9,70 \\
Atacama & $-7,07$ & 0,53 & 4,63 & 2,50 \\
Coquimbo & 0,49 & 0,22 & 2,44 & 2,82 \\
Valparaíso & 0,59 & 1,94 & 2,49 & 8,19 \\
RM & 1,85 & 2,50 & 1,98 & 42,24 \\
O’Higgins & 1,59 & 5,50 & 1,19 & 4,54 \\
Maule & 0,39 & 7,53 & 0,80 & 3,15 \\
Biobío & 3,31 & 0,97 & 2,12 & 7,11 \\
La Araucanía & $-0,02$ & 6,15 & 5,04 & 2,53 \\
Los Ríos & 3,71 & 2,13 & 2,83 & 1,29 \\
Los Lagos & 5,41 & 1,12 & 0,42 & 2,81 \\
Aysén & 5,15 & $-0,51$ & 3,61 & 0,54 \\
Magallanes & 2,37 & 3,88 & 4,12 & 1,06 \\
\hline Subtotal regionalizado & 1,91 & 2,30 & 1,59 & 91,51 \\
Extra-regional & $-0,06$ & 23,92 & 0,42 & 0,06 \\
IVA, derechos de importación & 1,92 & 1,60 & 1,56 & 8,42 \\
\hline PIB & 1,91 & 2,25 & 1,59 & 100 \\
\hline
\end{tabular}

Fuente: Elaboración propia en base a datos BC. 
C. Evolución Pobreza Multidimensional (4 dimensiones) por región, 2009-2015

\begin{tabular}{|l|c|c|c|c|c|}
\hline Regiones (de norte a sur) & 2009 & 2011 & 2013 & 2015 & 2009-2015 (var. \%) \\
\hline Arica y Parinacota & 24,6 & 27,1 & 23,6 & 21,0 & $-14,6$ \\
Tarapacá & 31,1 & 24,2 & 17,8 & 19,7 & $-36,7$ \\
Antofagasta & 30,5 & 25,0 & 20,7 & 16,0 & $-47,5$ \\
Atacama & 34,8 & 25,0 & 26,2 & 22,6 & $-35,1$ \\
Coquimbo & 30,7 & 25,1 & 21,6 & 23,0 & $-25,1$ \\
Valparaíso & 24,3 & 22,8 & 18,0 & 16,6 & $-31,7$ \\
RM & 24,8 & 20,0 & 18,0 & 18,4 & $-25,8$ \\
O’Higgins & 26,8 & 26,9 & 21,1 & 20,9 & $-22,0$ \\
Maule & 29,2 & 31,8 & 21,9 & 21,7 & $-25,7$ \\
Biobío & 28,2 & 28,7 & 22,4 & 17,2 & $-39,0$ \\
La Araucanía & 37,2 & 33,3 & 28,5 & 26,2 & $-29,6$ \\
Los Ríos & 29,8 & 25,3 & 22,9 & 20,1 & $-32,6$ \\
Los Lagos & 34,5 & 29,0 & 26,1 & 21,4 & $-38,0$ \\
Aysén & 32,4 & 26,9 & 22,3 & 15,7 & $-51,5$ \\
Magallanes & 18,3 & 13,6 & 11,8 & 9,1 & $-50,3$ \\
\hline Total nacional & $\mathbf{2 7 , 4}$ & $\mathbf{2 4 , 3}$ & $\mathbf{2 0 , 4}$ & $\mathbf{1 9 , 1}$ & $\mathbf{- 3 0 , 3 \%}$ \\
\hline
\end{tabular}

Fuente: Elaboración propia en base a Ministerio de Desarrollo Social (2015c).

\section{Resumen resultados socioeconómicos regionales}

\begin{tabular}{|c|c|c|c|c|c|c|}
\hline \multirow{2}{*}{ Variables (considera 15 regiones) } & \multicolumn{3}{|c|}{ Mejores } & \multicolumn{3}{|c|}{ Peores } \\
\hline & $1^{\circ}$ & $2^{\circ}$ & $3^{\circ}$ & $1^{\circ}$ & $2^{\circ}$ & $3^{\circ}$ \\
\hline PIB per cápita 2015 & Antofagasta & Atacama & Tarapacá & La Araucanía & $\begin{array}{c}\text { Arica y } \\
\text { Parinacota }\end{array}$ & Maule \\
\hline IDH 2003 & $\mathrm{RM}$ & Magallanes & Tarapacá & Maule & La Araucanía & Los Lagos \\
\hline IDERE (6 dimensiones) 2015 & $\mathrm{RM}$ & Magallanes & Antofagasta & La Araucanía & Maule & Aysén \\
\hline IDERE (7 dimensiones) 2015 & $\mathrm{RM}$ & Magallanes & Valparaíso & La Araucanía & Maule & Aysén \\
\hline Pobreza 2015 & Magallanes & Antofagasta & Aysén & La Araucanía & Maule & Biobío \\
\hline Pobreza Multidimensional 2015 & Magallanes & Aysén & Antofagasta & La Araucanía & Atacama & Coquimbo \\
\hline Indice 10/10 2015 & $\begin{array}{l}\text { Arica y } \\
\text { Parinacota }\end{array}$ & Antofagasta & Atacama & La Araucanía & Los Ríos & Los Lagos \\
\hline Indice $20 / 202015$ & $\begin{array}{c}\text { Arica y } \\
\text { Parinacota }\end{array}$ & Atacama & Antofagasta & La Araucanía & Los Ríos & Biobío \\
\hline Ingreso Autónomo del Hogar 2015 & RM & Antofagasta & Tarapacá & La Araucanía & Maule & Los Lagos \\
\hline Gini (met. nueva) 2015 & $\begin{array}{l}\text { Arica y } \\
\text { Parinacota }\end{array}$ & Atacama & Antofagasta & La Araucanía & $\mathrm{RM}$ & Los Ríos \\
\hline $\begin{array}{l}\text { Años de Escolaridad Promedio } \\
2015\end{array}$ & RM & $\begin{array}{l}\text { Tarapacá y } \\
\text { Antofagasta }\end{array}$ & $\begin{array}{c}\text { Arica y } \\
\text { Parinacota }\end{array}$ & $\begin{array}{l}\text { Maule y Los } \\
\text { Lagos }\end{array}$ & La Araucanía & $\begin{array}{l}\text { O’Higgins y } \\
\text { Los Ríos }\end{array}$ \\
\hline$\%$ Analfabetismo 2015 & Tarapacá & Magallanes & Antofagasta & Maule & La Araucanía & Los Ríos \\
\hline Esperanza de Vida 2015 & Coquimbo & $\mathrm{RM}$ & Atacama & Los Lagos & Antofagasta & Magallanes \\
\hline Especialistas/10.000 hab. 2015 & RM & Magallanes & Aysén & Maule & Atacama & Tarapacá \\
\hline
\end{tabular}

Fuente: Elaboración propia. 


\section{E. Definiciones}

Años Escolaridad Promedio: Años de escolaridad personas con 15 años o más. Obtenido de la Encuesta CASEN.

Aporte Sector Primario al PIB 2009: Aporte al PIB regional, de los sectores silvoagropecuario, pesca y minería. Calculado con base en datos Banco Central de Chile.

Aporte Sector Secundario al PIB 2009: Aporte al PIB regional, de los sectores industria manufacturera; electricidad, agua y gas y sector construcción. Calculado con base en datos Banco Central de Chile.

Aporte Sector Terciario al PIB 2009: Aporte al PIB regional, de los sectores comercio, restaurantes y hoteles; transporte, información y comunicaciones; servicios financieros y empresariales; servicios de vivienda e inmobiliarios; servicios personales y administración pública, 2009. Calculado con base en datos Banco Central de Chile.

Esperanza de Vida al nacer: Años promedio de esperanza de vida al nacer. Obtenido de INE Chile.

Indice de Gini: "El índice de Gini mide hasta qué punto la distribución del ingreso (o, en algunos casos, el gasto de consumo) entre individuos u hogares dentro de una economía se aleja de una distribución perfectamente equitativa". "Un índice de Gini de 0 representa una equidad perfecta, mientras que un índice de 100 representa una inequidad perfecta" (Banco Mundial, 2018). Obtenido de Resultados Encuesta CASEN (años 2013 y 2015 calculado por el autor).

Ingreso Autónomo del Hogar: "Es la suma de todos los pagos que reciben todos los miembros del hogar, excluido el servicio doméstico puertas adentro, provenientes tanto del trabajo como de la propiedad de los activos. Estos incluyen sueldos y salarios, monetarios y en especies, ganancias provenientes del trabajo independiente, la autoprovisión de bienes producidos por el hogar, rentas, intereses, dividendos y retiro de utilidades, jubilaciones, pensiones o montepíos, y transferencias corrientes" (Ministerio de Desarrollo Social, 2015c, pp. 6). Obtenido de la Encuesta CASEN.

Ingreso Primer Quintil: Corresponde al ingreso autónomo del hogar del 20\% de la población de menores ingresos. Calculado con base en la Encuesta CASEN.

Ingreso Último Quintil: Corresponde al ingreso autónomo del hogar del 20\% de la población de mayores ingresos. Calculado con base en la Encuesta CASEN. 
PIB per cápita: Es el Producto Interno Bruto por cada habitante. Obtenido con base en el Banco Central de Chile (2018). Se calculó utilizando el método de la tasa de variación, de forma tal de unir una serie de datos del PIB por región, a precios constantes, base 1986, 1996, 2003 y PIB, volumen a precios del año anterior encadenado, series empalmadas, referencia 2013, llevando los valores a este último año.

Ratio 20/20: "Índice de desigualdad que muestra la relación entre el ingreso recibido por el 20\% de hogares de mayores ingresos y el correspondiente al 20\% de hogares con menores ingresos" (Ministerio de Desarrollo Social, 2015c, pp. 37). Calculado con base en la Encuesta CASEN.

Tasa Bruta de Mortalidad (TBM): "Es la cantidad de muertes en un año, por cada 1000 habitantes, estimada a mitad de año" (Banco Mundial, 2018).

Tasa Bruta de Natalidad (TBN): "Indica la cantidad de nacidos vivos en el año, por cada 1000 habitantes, estimada a mitad de año" (Banco Mundial, 2018).

Tasa de Fertilidad: "La tasa de fertilidad total representa la cantidad de hijos que tendría una mujer si viviera hasta el final de sus años de fertilidad y tuviera hijos de acuerdo con las tasas de fertilidad actuales específicas por edad" (Banco Mundial, 2018).

Tasa Bruta de Mortalidad Infantil: Es la cantidad de infantes que mueren antes de llegar al año de vida, por cada 1000 nacidos vivos, en un año determinado (Banco Mundial, 2018).

Tasa Generacional de Reemplazo: "Es la medida que indica si una población está reemplazándose a sí misma de forma efectiva o no. Por lo general hace falta que cada mujer en edad fértil tenga entre 2,2 y 2,5 hijos para que dicho reemplazo generacional se produzca", extraído de http://glosarios.servidor-alicante.com/geografia-humana/ tasa-de-reemplazo-generacional. 
